\title{
Análisis de la elección modal de transporte público y privado en la ciudad de Popayán
}

Analysis of the Modal Choice of Public and Private Transport in the City of Popayan

\section{Análise da Escolba Modal de Transporte Público e Privado na Cidade de Popayán}

\author{
Claudia Liceth Fajardo Hoyos* \\ Andrés Mauricio Gómez Sánchez ${ }^{* \star}$
}

Recibido: 1 de septiembre de 2014

Aprobado: 24 de abril de 2015

Doi: dx.doi.org/10.12804/territ33.2015.07

Para citar este artículo:

Fajardo, H. C. L., \& Gómez, S. A. M. (2015). Análisis de la elección modal de transporte público y privado en la ciudad de Popayán. Territorios, 33, 157-190. doi: dx.doi.org/10.12804/territ33.2015.07

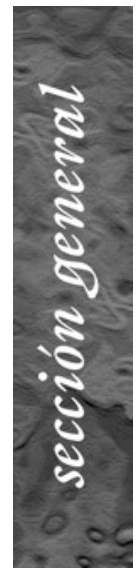

* Docente ocasional tiempo completo, Departamento de Economía, Unipersidad del Canca, Popayán, Colombia. Miembro del Grupo de investigación Entropia, Universidad del Canca. Economista, Universidad del Valle, Cali, Colombia. Magister en Economía Aplicada de la Universidad del Valle. Correo electrónico: cfajardo@ unicauca.edu.co

** Docente titular, Departamento de Economía, Universidad del Cauca, Popayán, Colombia. Miembro del Grupo de investigación Entropia, Universidad del Canca. Economista, Universidad del Valle, Cali, Colombia. Especialista, Gerencia de Proyectos, Universidad del Cauca, Popayán, Colombia. Magister en Economía Aplicada, Universidad del Valle, Cali, Colombia. Correo electrónico: amgomez@ unicauca.edu.co. 
Palabras clave

Demanda de transporte, utilidad aleatoria, transporte público, transporte privado, Logit Multinomial.

Keywords

Transport demand, random utility, public

transport, private transport, Multinomial Logit.

Palavras-chave

Demanda de transporte, utilidade aleatória, transporte público, transporte privado, logit multinomial.

territarias 33

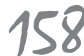

\section{RESUMEN}

El objetivo de este estudio es analizar la elección que hacen los payaneses entre siete modalidades diferentes de transporte, tanto público como privado. La elección se basa en la teoría del consumidor, a partir de una función de utilidad indirecta y aleatoria, la cual considera qué aspectos, tanto sociales como económicos de los demandantes, influyen de manera directa en dicho proceso. Para capturar la elección se utilizan dos modelos econométricos del tipo Logit Multinomial. Los resultados, entre muchos otros, evidencian que los costos, el tiempo, los ingresos y la edad se constituyen en factores determinantes en la elección modal de transporte en la ciudad de Popayán, a diferencia de variables como el género y/o ser jefe de hogar que no resultan ser relevantes.

\section{ABSTRACT}

The aim of this study is to analyze the choice people do seven different modes of both public and private transport in Popayán. The choice is based on consumer theory from a random function and indirect utility, which considers both social and economic aspects of the plaintiffs have a direct influence on the process. The method used to capture the choice is a multinomial logit econometric model. The results among many others, show that the costs, time, income and age are determining factors in the choice of modal transport in the city of Popayan, unlike variables such as genre being head of household, the which are not relevant.

\section{RESUMO}

O objetivo deste estudo é analisar a escolha que fazem os habitantes de Popayán entre sete modalidades diferentes de transporte tanto público quanto privado. A escolha baseia-se na teoria do consumidor a partir de uma função de utilidade indireta e aleatória, a qual considera que aspetos tanto sociais quanto económicos dos demandantes influem de forma direta em dito processo. $\mathrm{O}$ método utilizado para capturar a escolha são dois modelos econométricos do tipo Logit Multinomial. Os resultados entre muitos outros, evidenciam que os custos, o tempo, os ingressos e a idade se constituem em fatores determinantes na escolha modal de transporte na cidade de Popayán, a diferença de variáveis como o gênero e/ou ser chefe da família, as quais não resultam ser relevantes. 


\section{Introducción}

La necesidad de transportarse en y hacia países, ciudades y regiones ha sido una prioridad para los individuos, desde que las distancias entre ellos y sus lugares de destino se han ido ampliando cada vez más. La búsqueda de diferentes tipos de transporte más eficaces y eficientes ha llevado a la implementación de sistemas de movilidad, tanto públicos como privados para lograr una mayor calidad en el servicio.

Estos sistemas de transporte, siguiendo a Rodrigue (2013), están estrechamente relacionados con los cambios socioeconómicos de los países, a partir de la revolución industrial, toda vez que la movilidad de las personas, mercancías y los niveles de accesibilidad territorial son el núcleo de esta relación. El buen funcionamiento de estos ha permitido que las personas puedan desplazarse de un lugar a otro por diferentes motivos (trabajo, estudio, recreación, deporte, ocio, entre otros), lo que ayuda también a distribuir regionalmente la población, las industrias y los ingresos (Causado, 2009). Por tal razón, los sistemas de transporte son un asunto interés público y, por tanto, requieren regulación por medio de leyes y políticas públicas que faciliten su control y adecuado funcionamiento (Roa, 2009). En este sentido, las ciudades más pobladas del mundo, y también las de Colombia, han determinado implementar sistemas masivos de transporte para poder controlar y planear la movilidad de sus ciudadanos. Una adecuada planeación en esta materia le permite al Estado tomar decisiones más acertadas para la inversión en infraestructura, que resuelvan las necesidades de accesibilidad, conectividad y articulación urbana y regional.

En este sentido, el Gobierno nacional, consciente de la necesidad de mejorar la movilidad, formuló, no solo en las ciudades grandes del país sino también en las ciudades intermedias, el Programa Nacional de Transporte Urbano, ${ }^{1}$ el cual busca, básicamente, impulsar la implantación de sistemas integrados de transporte masivo y fortalecer la capacidad institucional para planear y gestionar el tráfico y transporte en las demás ciudades. En dicho documento se estableció desarrollar el "Sistema Estratégico de Transporte Público de Pasajeros" (SETP) para varias ciudades del país donde se incluyó a la ciudad de Popayán, iniciativa que fue recibida con gran expectativa a nivel local, ya que esta ciudad presenta diferentes problemas de movilidad. ${ }^{2}$

Los principales problemas de Popayán se originan por la existencia de un sistema de transporte público colectivo ineficiente (ya que es lento y costoso), cuya sobreoferta es cercana al $60 \%$. El esquema institucional que opera en el transporte urbano no es el mejor, pues está segmentado, es decir, el servicio está en manos de tres agentes (las empresas afiliadoras, los propietarios de los vehículos y los conductores), quienes tienen intereses particulares y diferentes, situación que genera un sistema de recaudo independiente. Todos lo observan como un negocio particular, lo que va en detrimento de la operación y la calidad del servicio que se le presta al usuario. A esto se le suman los
${ }^{1}$ Documento Compes 3167 y 3602 de 2002.

2 Popayán es una ciudad ubicada al suroccidente de Colombia, y es la capital del Departamento del Canca. La ciudad está dividida en nueve comunas, las cuales albergan alrededor de 270000 habitantes ( $88 \%$ espoblación urbana) (ORMET, 2013). Su economía se basa en el sector comercialyen el de servicios, con una baja participación del sector industrial. Es reconocida por la preservación de su arquitectura colonial y sus festividades de carácter religioso.

territarias 33

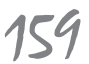


sistemas de transporte ilegales, que cada vez toman más fuerza en la ciudad (mototaxis), los cuales, por su condición, no ofrecen ningún tipo de garantía de seguridad para el usuario, ni tarifas reguladas.

Desde la esfera de las economías capitalistas, los problemas del transporte público y privado, en cualquier contexto geográfico, deben analizarse no solo a partir de la oferta del servicio sino también desde la demanda, ya que es un mercado como cualquier otro. Los estudios existentes a nivel nacional e internacional, desde ambas perspectivas, son diversos, pero a nivel local son inexistentes desde el lado de la demanda.

En este orden de ideas, el presente documento intenta generar indicios del comportamiento de la demanda, y no de la oferta, para esta ciudad, como una forma de contribuir al análisis y solución de la problemática actual, por medio del estudio de las preferencias de elección modal entre diferentes alternativas de transporte para los payaneses, controladas por un conjunto de características socioeconómicas. Hasta el momento, el único trabajo que se conoce en la ciudad frente a este tema fue realizado por la Alcaldía de Popayán (2008), el cual estudia, solo desde la oferta, el transporte en un solo medio (colectivo) y deja por fuera a otros medios (públicos y privados). En este se realiza un análisis de carácter netamente descriptivo, sin tener en cuenta la demanda y las posibles relaciones de causalidad que podrían estar influyendo en el proceso de elección entre diferentes tipos de transporte.

Las modalidades de transporte ex- múnmente aceptadas y las utilizadas en el ámbito local: a pie, bicicleta, moto particular, mototaxi, colectivo, taxi y automóvil. Debido a la carencia de información, se implementó una encuesta por medio de un muestreo aleatorio incidental. Como hipótesis de partida, se espera que las características socioeconómicas de los individuos expliquen la elección modal, en especial las variables que hacen referencia al tiempo por desplazamiento y los costos. Así mismo, el ingreso se considera clave, puesto que el transporte hace parte importante de los gastos familiares, pues ingresos mayores pueden permitir mayores usos de algunos medios o el uso de medios más costosos. El tema propuesto se desarrollará en cuatro secciones, la primera es esta introducción. La segunda sección contiene una revisión de los principales referentes teóricos y algunos estudios de la demanda de transporte para diferentes ciudades de Colombia y para Popayán. Posteriormente, se expone la metodología, los modelos econométricos y los resultados de las estimaciones de los modelos Logit multinomiales. Por último, en la sección cuarta, se presentan las conclusiones del estudio y algunas recomendaciones de política.

\section{Referentes teóricos y estudios aplicados}

\subsection{Referentes teóricos}

La modelización de la demanda de transporte se ha basado, tradicionalmente, en el empleo de dos tipos de modelos: los mo- 
delos agregados o de primera generación y los modelos desagregados o de segunda generación. Los primeros fueron utilizados en su mayoría en los estudios de transporte hasta finales de los años setenta. Por su parte, los modelos desagregados comienzan a ser populares en los años ochenta, por el hecho de que presentan ciertas ventajas sobre los modelos usados hasta el momento. Este tipo de modelos se basan en el uso de los datos a nivel individual, lo que permite una mejor comprensión de los comportamientos de viaje, puesto que se basan en las teorías de la elección individual.

La demanda de transporte puede definirse como la disposición a pagar, que tienen los consumidores, por hacer uso de una determinada infraestructura o servicio de transporte; así mismo, se puede definir como la cantidad de servicios y usos de las infraestructuras que se desean comprar a cada precio (De Rus, Campos y Nombela, 2004). El precio del servicio está inversamente relacionado con la cantidad demandada de viajes. Si el ingreso de los habitantes de una región aumenta de manera permanente y no ocasional, la demanda de transporte aumentará, ya sea en cantidad de viajes o de kilómetros recorridos (Mendieta \& Perdomo, 2008). Dada la información disponible, la demanda de transporte puede especificarse en función de diferentes variables explicativas, según sea una demanda individual o agregada. Otras variables determinantes en la explicación de la demanda de transporte son la velocidad, la calidad, frecuencia estándar, comodidad, confiabilidad y seguridad del servicio.
Los modelos de demanda de transporte se enmarcan en los enfoques de las preferencias reveladas, preferencias declaradas o una combinación de ambas. En el caso en el que se tiene información de los viajes realizados por los usuarios y del tiempo invertido en el viaje, la estimación de la demanda se puede modelar a partir de la teoría de dualidad del consumidor. A este enfoque se le conoce con el nombre de preferencias reveladas. En el caso en el que un usuario solo puede decir si estaría dispuesto a viajar en un determinado medio de transporte, la estimación y especificación de la demanda requiere de modelos probabilísticos, este enfoque se conoce como el enfoque de preferencias declaradas. En los casos en los que se requiere estimar sistemas de demanda de transporte, en los que se tienen algunos medios de transporte que están en funcionamiento y otros que aún no, se puede usar información procedente del enfoque de preferencias reveladas e información procedente de preferencias declaradas.

En el enfoque de preferencias reveladas, la estimación se hace por medio del modelo de elección individual, donde se obtiene una demanda marshalliana, cuyas dos variables explicativas son el precio generalizado y el ingreso del usuario. En el enfoque de preferencias declaradas se puede modelar la demanda por un medio de transporte, a partir de la estimación de la probabilidad de elegir ese medio. Este modelo, conocido como modelo de utilidad aleatoria, implica una decisión excluyente por parte de los usuarios, es decir, nadie va en bus y carro particular al mismo tiempo. territarias 33

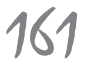




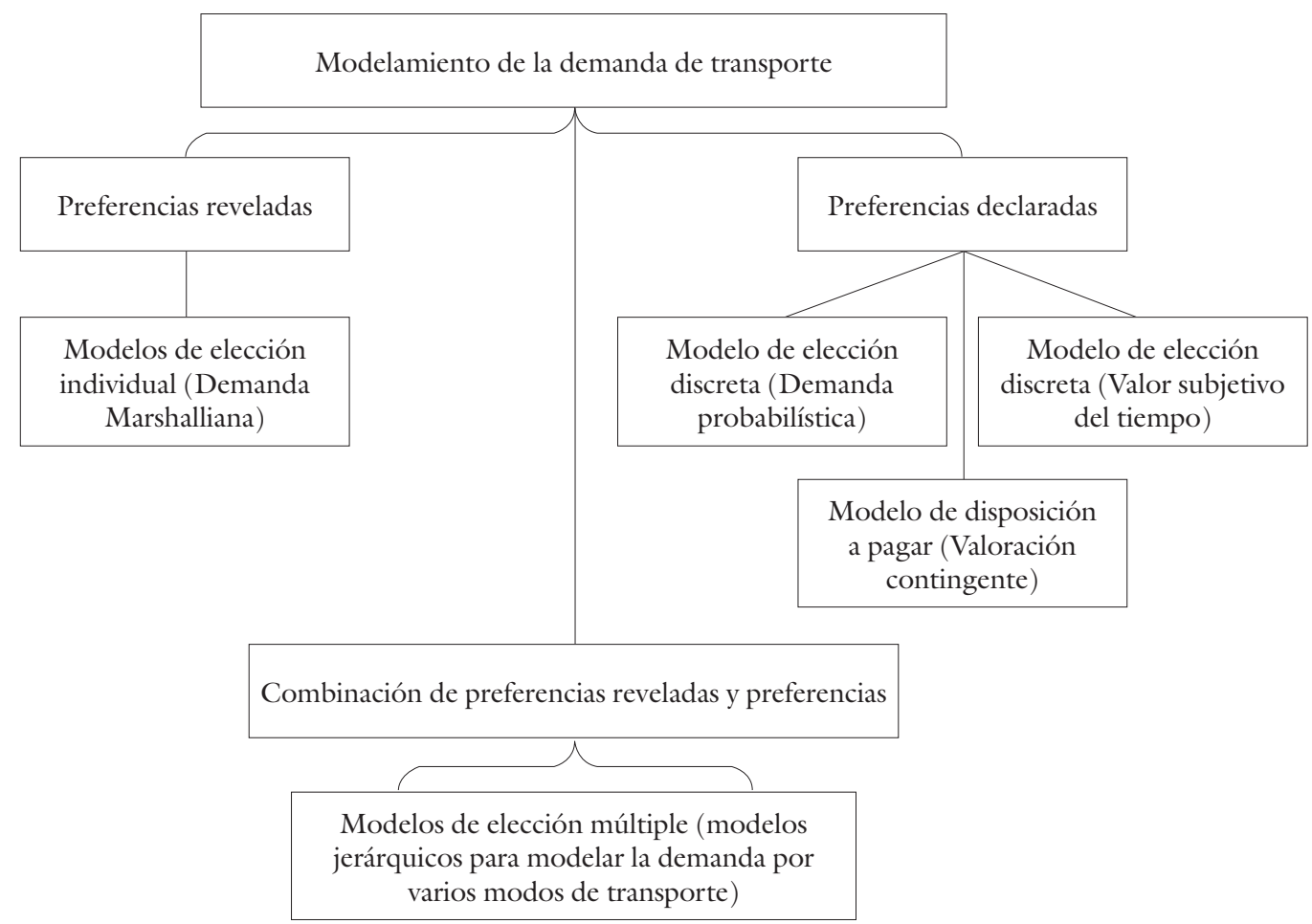

Fuente: Mendieta y Perdomo (2008).

Con los modelos de elección discreta también se puede estimar el valor subjetivo del tiempo. Una vez estimados los parámetros de la función de utilidad indirecta, se puede calcular la tasa marginal de sustitución entre las utilidades marginales del tiempo de viaje y el costo de este. Otros modelos, basados en la teoría de la utilidad aleatoria, son los modelos de disposición a pagar, utilizados para estimar la tarifa que tendrá un nuevo medio de transporte. Finalmente, el modelo multimodal o jerárquico se caracteriza porque ahora el usuario puede elegir transportarse en más de dos medios o puede elegir usar una vía dentro de un conjunto de posibilidades disponibles (Mendieta \& Perdomo, 2008).

La ciudad de Popayán no cuenta con una base de datos que revele el número de viajes realizados por individuo ni el tiempo empleado en cada uno de ellos, por tal razón no es pertinente hacer uso de la teoría dual del consumidor. Por el contrario, se pretende indagar a los usuarios acerca de cuál es el medio que eligen con más frecuencia, esto ubica en el campo de las 
preferencias declaradas y en la teoría de la utilidad aleatoria, ya que el individuo deberá elegir solo un modo entre siete diferentes. Entonces, este análisis de la demanda de transporte de los payaneses se enmarca, claramente, en los modelos de elección discreta.

\subsubsection{Preferencias declaradas: el modelo de elección discreta}

En los casos en que se carece de información que revele las preferencias de los usuarios por un medio de transporte, la variable dependiente, relacionada con el número de viajes realizados en un periodo, se reemplaza por la elección de dicho medio de transporte. Esta decisión se conoce con el nombre de elección discreta. Estos modelos describen la decisión de elección de entre un conjunto de alternativas disponibles, es decir, si existen tres opciones de transporte: carro, autobús y motocicleta, el individuo puede decidir en cuál opción desea realizar su viaje. Los que toman las decisiones pueden ser personas, hogares, firmas, y cualquier otra unidad de decisión (Train, 2003 citado en Mendieta \& Perdomo, 2008). Para el caso específico de la ciudad de Popayán, la elección posible de los agentes se hace entre siete diferentes categorías: a pie, bicicleta, moto particular, mototaxis, colectivos, taxis, y automóvil.

El análisis de elección discreta usa el principio de maximización de utilidad, y supone que el individuo, al tomar su decisión sobre la elección de un determinado medio de transporte dentro de un conjunto de alternativas disponibles, está eligiendo también alcanzar su máximo nivel de utilidad. La elección de carácter discreto se puede explicar con la teoría de la elección del consumidor, basada en la función de utilidad indirecta. Además, es necesario hacer uso del modelo de utilidad aleatoria para explicar por qué un usuario elige un medio de transporte y no otro (Mendieta \& Perdomo, 2008). La función de utilidad de un individuo se encuentra especificada como:

$$
U_{i j}\left(X_{i j}, S ; \beta\right)
$$

Donde $X_{i j}$ representa el conjunto de atributos implícitos en los medios de transporte; $S$ denota un conjunto de características del individuo que toma la decisión, y $\beta$ representa un conjunto de parámetros no conocidos. Esta función indirecta de utilidad se divide en dos componentes, uno determinístico y otro aleatorio, así:

$$
U_{i j}\left(X_{i j}, S ; \beta\right)=V_{i j} U_{i j}\left(X_{i j}, S ; \beta\right)+\varepsilon_{i j}
$$

La probabilidad de viajar en el medio de transporte $j$ para el individuo $i$, es equivalente a la probabilidad de que la utilidad indirecta que le proporciona dicho medio $\left(V_{i j}\right)$ sea superior a la de cualquier otro $\left(V_{i o}\right)$ :

$$
\begin{aligned}
& \operatorname{Prob}(j)=\operatorname{Prob}\left[\left(V_{i j}\left(X_{i j}, S ; \beta\right)+\varepsilon_{i j}\right)>\right. \\
& \left.\left(V_{i o}\left(X_{i o}, S ; \beta\right)+\varepsilon_{o}\right)\right]=\operatorname{Prob}\left[\varepsilon_{o}+\varepsilon_{i j}\right. \\
& \left.<V_{j}(.)-V_{o}(.)\right]
\end{aligned}
$$


La expresión anterior presenta la elección modal en términos de la distribución de probabilidad. Por lo general, dependiendo de la distribución que se asigne al término aleatorio, los modelos econométricos que se pueden utilizar son los Logit o los Probit. Si la decisión del medio de transporte se hace de manera secuencial, se utilizan los modelos Logit jerárquicos (Vivas, 2009). Los modelos de elección discreta han sido ampliamente utilizados en la literatura económica de los últimos años, especialmente en la economía del transporte. Estos modelos permiten realizar una estimación cuando la variable dependiente solo puede tomar un número finito de valores, siendo esta variable una probabilidad no observada, y las observaciones elecciones individuales. Aunque los modelos desagregados pueden aplicarse en todas las áreas de planificación del transporte, ha sido una tendencia habitual el centrarse en modelos de elección del modo de transporte.

Para los modelos de elección discreta, según el número de alternativas incluidas en la variable endógena, se distinguen los modelos de respuesta dicotómica frente a los denominados modelos de respuesta o elección múltiple. Según la función utilizada para la estimación de la probabilidad, existen el modelo de probabilidad lineal truncado, el modelo Logity el modelo Probit. Según que las alternativas de la variable endógena, sean excluyentes o incorporen información ordinal, se distinguen entre los modelos con datos no ordenados y los modelos con datos ordenados. Dentro de los primeros, según si los regresores hacen referencia a aspectos específicos de la muestra o de las alternativas entre las que se ha de elegir, se distingue entre los modelos multinomiales y los condicionales (Medina, 2003).

La elección entre siete diferentes categorías implica la utilización de modelos de elección discreta de respuesta múltiple con datos no ordenados; así mismo, la relación de la elección con las características de quien elige, implicaría la utilización de un modelo multinomial o anidado. Sin embargo, para el caso de Popayán, en un ejercicio previo se observó que es el Logit multinomial el modelo que mejor se ajusta a la explicación de la demanda de transporte. El principal supuesto del modelo Logit es que los términos aleatorios de la función de utilidad son independientes e idénticamente distribuidos (IID), lo cual implica que los individuos con las mismas características observables tienen gustos idénticos, y que cualquier efecto derivado de las características no observables de los individuos o las alternativas no están correlacionados por medio de los individuos ni de las alternativas. MacFadden (1974) ha demostrado que las correspondientes probabilidades de un modelo multinomial con término de error que sigue esa distribución son:

$$
P_{i j}=e^{V_{j}} / \sum_{j=1}^{j} e^{V_{j}}
$$

\section{territarias 33} 164 
Esta es la forma general de la función de distribución logística, y expresa la probabilidad de que un individuo $i$ escoja la alternativa $j$. Las ecuaciones estimadas proporcionan un conjunto de probabilidades para las $J+1$, alternativas que puede elegir una persona, que tenga $x_{i}$ como características individuales. Es importante eliminar una indeterminación que presenta el modelo. $\mathrm{Si}$ se define $\beta^{\prime}{ }_{j}=\beta_{j}+q$ para cualquier vector $q$, se obtienen exactamente las mismas probabilidades, puesto que todos los términos a los que q afectan se cancelan. Para resolver este problema, se puede normalizar el modelo tomando $\beta_{o}=0$ (Greene, 1999). Por lo tanto las probabilidades resultantes son:

$$
V_{i j}=\sum_{l} \beta_{j l} X_{j l}
$$

En este caso, se supone que los parámetros $\beta$ son constantes para todos los individuos, aun cuando pueden variar entre alternativas, donde $l$ refleja el número de atributos que entran en la función de utilidad de cada alternativa. Además, para una opción cualquiera $j$ existe en $X_{i j}$ que toma el valor uno para todos los individuos que tienen disponible esa alternativa; el coeficiente $\beta_{j l}$ correspondiente a esa variable se interpreta como constante modal específica de la alternativa en cuestión, en este caso de $j$.

$$
\begin{gathered}
\operatorname{Prob}(\Upsilon=j)=e^{\beta^{\prime}{ }_{j} X_{i}} / 1+\sum_{k=1}^{j} e^{\beta^{\prime}{ }_{j} X_{i}} \quad j=1,2, \cdots, J \\
\operatorname{Prob}(\Upsilon=j)=1 / 1+\sum_{k=1}^{j} e^{\beta^{\prime}{ }_{j} X_{i}}
\end{gathered}
$$

Así, si, por ejemplo, se consideran tres alternativas (denotadas por 1, 2 y 3), esta probabilidad también puede expresarse en forma de comparaciones binarias, tal y como se hacía en el Logit binomial, expresando la probabilidad de elegir la alternativa 1 como:

$$
P_{i j}=1 /\left(1+e^{V i 2-V i l}+e^{V i 2-V i l}\right)
$$

Normalmente, se considera que las funciones de utilidad son lineales en los parámetros, en cuyo caso vendrían dados por una expresión como la que sigue:
Como el modelo funciona con base en diferencias, no es posible especificar una constante para cada alternativa, por el contrario, lo que se hace es especificar una de las constantes como referencia (igual a cero) y las restantes se obtienen de la estimación, por lo que su valor es relativo al que toma la constante de referencia. En el modelo Logit multinomial, las variables independientes pueden ser incluidas de dos maneras. La primera consiste en establecer los coeficientes de una variable como específica para cada una de las alternativas, así cada alternativa o medio de transporte tendrá una estimación propia. También se 
${ }^{3}$ Se habla de preferencias reveladas cuando se observa el comportamiento real de los usuarios, por ejemplo, el medio de transporte utilizado, $y$ de preferencias declaradas o establecidas cuando se obtienen respuestas de los individuos ante situaciones de elección hipotéticas. Finalmente, los datos mixtos contienen información proveniente de preferencias reveladas y de preferencias declaradas. pueden especificar los coeficientes de las variables genéricamente para todos los medios, y así el coeficiente estimado será uno solo para todos los medios de transporte (Mendieta \& Perdomo, 2008).

Para estimar los parámetros de la función de utilidad en el modelo Logit multinomial se utiliza, en la mayoría de los casos, el método de máxima verosimilitud, y se usa información acerca de preferencias reveladas o declaradas por los usuarios. ${ }^{3} \mathrm{El}$ método de máxima verosimilitud se basa en la idea de que, aunque un ejemplo podría ser originado a partir de muchas poblaciones, existe una probabilidad mayor de que haya sido extraído de una determinada población más que de otra. El método de máxima verosimilitud estima el conjunto de parámetros que con mayor probabilidad generará el ejemplo objeto de estudio. Para una muestra aleatoria de tamaño $\mathrm{Nla}$ función de verosimilitud se puede expresar como el producto de las probabilidades de elección asociadas con $M$ subconjuntos de observaciones, en el que el primer subconjunto incluye $N_{1}$ individuos que han elegido la alternativa 1; el próximo subconjunto contiene $\mathrm{N}_{2}$ individuos que han elegido la alternativa 2, y así sucesivamente, donde todas las observaciones son independientes:

$$
L(\beta)=\prod_{i=1}^{N_{1}} P_{i l} \prod_{i=N_{1}+1}^{N_{1}+N_{2}} P_{i 2} \ldots \prod_{i=N_{1}+N_{2}+\cdots+N_{M-1}+1}^{N_{1}+N_{2}} P_{i l}
$$

territarios 33 166 ne el valor de 1 cuando el individuo 1 elige la alternativa $j$-que pertenece al conjunto de alternativas que tienen disponible $\left(C_{i j}\right)$ y cero en el resto de los casos-.

$$
L(\beta)=\prod_{i=1}^{N} \prod_{j \in C_{i}} P_{i j}^{d_{i j}}
$$

Para simplificar dicha expresión, se aplica logaritmo sin que se alteren los resultados de la maximización, ya que este coincide para una función dada y el logaritmo de esta:

$$
\operatorname{Ln} L=l(\beta)=\sum_{i=1}^{N} \sum_{j \in C_{i}} d_{i j} \ln P_{i j}
$$

$\mathrm{Al}$ maximizar se obtiene un conjunto de parámetros estimados que se distribuyen asintóticamente Normal con media el valor real de dichos parámetros y varianza la matriz de las segunda derivadas de la función de verosimilitud (González, 1995).

\subsection{Estudios aplicados}

A nivel internacional, un esfuerzo a referenciar es el realizado por Martín y Román (1999), en el cual se intenta analizar y predecir la demanda de los sistemas de transporte. Se enfatiza en cuáles son los principales factores que determinan la demanda de transporte de mercancías y pasajeros, y se realiza una revisión de los enfoques me- 
todológicos más usuales para predecir la demanda. También se analiza la evolución experimentada por la demanda de transporte, pasajeros y mercancías en España en los últimos años y se detecta cómo algunos de los factores determinantes han dado lugar a una tendencia hacia la sustitución de unos modos por otros. En otro estudio, Galán (2005) determina la demanda de transporte público y privado para la ciudad de Monterrey, en México. El autor implementa un conjunto de modelos econométricos del tipo Probit binario para capturar el comportamiento de los individuos sobre el medio que utilizarán para realizar sus viajes. Los resultados muestran que las variables densidad poblacional, distancia y edad no son relevantes, pero otras como el tiempo, costo y escolaridad son claves a la hora de explicar dicha demanda.

Los estudios realizados a nivel nacional han sido diversos en lo corrido del nuevo milenio. En un primer esfuerzo, Alvis, Arellano \& Toro (2005) realizan una aplicación del modelo Probit para el caso de la ciudad de Cartagena, donde encuentran que con base en las preferencias de los usuarios, se deberán tener en cuenta tres elementos clave: disponibilidad, precio y rapidez, pues estas variables son las que, en general, determinan la decisión de los usuarios sobre cuál medio de transporte usar para desplazarse hacia su lugar de estudio o trabajo.

Para Bogotá, se encuentra el estudio realizado por Chavarriaga (2006). Esta investigación busca conocer los determinantes de los diferentes medios de transporte público en Bogotá, y mostrar cómo influ- yen las características de los ciudadanos en la toma de decisiones, al enfrentarse a los diferentes medios de transporte existentes en la ciudad. Para tal efecto se utiliza un modelo Logit multinomial (MLM). De otro lado, el estudio de Moreno, Sarmiento \& González (2010) tiene por objetivo evaluar las políticas para influir en la elección modal de usuarios de vehículo privado en universidades, con caso específico de la Universidad de Antioquia. Esta investigación estimó tanto un modelo logit multinomial como un logit jerárquico, con datos provenientes de encuestas realizadas bajo las técnicas, tanto de preferencias reveladas como de preferencias declaradas. El modelo que mejores resultados arrojó en este caso fue el modelo anidado. Finalmente, Moreno et al. (2010), que estudiaron la elección del modo de transporte para los viajes desde y hasta la Universidad de Antioquia. De la misma manera, los costos y los tiempos de viaje determinan la elección modal. La aplicación del modelo anidado requiere, en muchos casos, de la utilización de datos provenientes de encuestas, tanto de preferencias reveladas como de preferencias declaradas.

Aunque no existen datos para Popayán en materia de elección de transporte, es decir desde la demanda, el único estudio que se encuentra desde la oferta fue realizado por la Alcaldía Municipal para el año 2008. Aquí, se revela que frente al sistema de transporte de la ciudad, los medios más utilizados son los de carácter público formal, como los colectivos, busetones, buses y taxis; los de carácter público informal, territarias 33

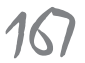


${ }^{4}$ Estos trestipos de transporte se pueden agrupar, puesto que el número de buses es muy reducido y solo cubre tres rutas en la ciudad, $y$ el busetón y el colectivo son vehiculos de características similares y cubren rutas indistintamente.

5 Transportes Pubenza, Transportadora Libertad, Sotracauca y TransTambo.

- Servitaxi (327), Tax Belalcázar (585), Trans Tambo (128) y Trans Andino (86).

7 Decreto N. 00532 de 2009, Alcaldía de Popayán, Secretaria de Tránsito y Transporte.

\section{tersitarias 33} 168 como los mototaxis; y los privados, como el automóvil, las motos y las bicicletas, y, finalmente, el modo a pie. Dentro de la categoría transporte público, se puede agrupar a los colectivos, buses y busetones en un solo modo de trasporte. ${ }^{4}$ Este servicio es ofrecido por cuatro empresas ${ }^{5}$ que cuentan con 47 rutas autorizadas y con un parque automotor de 663 vehículos. Estos vehículos, en promedio, transportan por día 101604 pasajeros (ICER Cauca, 2012). Solo el $49 \%$ de las rutas de transporte público colectivo tienen una ocupación del 100\% en las horas pico. Este sistema tiene un alto grado de superposición, cada ruta tiene en promedio una frecuencia de despacho de tres minutos y un recorrido de $45 \mathrm{~km}$, que es un porcentaje alto cuando se compara con el promedio nacional, que está en 20 $\mathrm{km}$ (CONPES, 2009). Finalmente, la tarifa de este modo ha crecido a una tasa promedio de $8,3 \%$ anual, en términos reales, en los últimos dos años.

De otro lado, el estudio identifica que el esquema institucional que opera en el transporte urbano en el municipio de Popayán no es el más adecuado, ya que la responsabilidad de la operación se diluye entre las empresas afiliadoras, los conductores y los propietarios. Las empresas son intermediarias entre la autoridad y los propietarios, que en general son un gran número, debido a la dispersión de la propiedad del parque automotor. Los propietarios son inversionistas que ven en el vehículo un negocio independiente, esto cumple un papel fundamental en la operación y en la calidad del servicio que se presta al usuario. Como los mismos conductores son los responsables del recaudo del sistema, esto genera lo que se conoce en el lenguaje popular como “la guerra del centavo" (CONPES, 2009).

Otro medio de transporte público sobre el que se hace énfasis en la investigación, es el servicio de taxi. Dicho servicio es prestado por cuatro empresas, ${ }^{6}$ con un parque automotor de 1126 vehículos. El cobro de las carreras se hace teniendo en cuenta la zona donde el pasajero abordó el vehículo y la zona donde termina la carrera, cada zona tiene su propia tarifa y los desplazamientos en la misma zona se cobran con la tarifa mínima. Dicha tarifa también cambia según la hora y el día (si es festivo u ordinario). ${ }^{7}$ Popayán, así como otras ciudades intermedias de Colombia, tiene un transporte público informal denominado mototaxi. Actualmente, la Alcaldía de Popayán tiene solo 41 motos públicas registradas. La figura de mototaxi no está autorizada para matricular como tal, es por eso que la Secretaría de Tránsito y Transporte no tiene registros al respecto. La informalidad en este tipo de actividad trae consigo serios problemas, además de los propios de la inclusión de la fuerza de trabajo al sistema de seguridad social, como son la inseguridad y la falta de protección de los pasajeros ante un eventual accidente.

El estudio también muestra que algunas familias solucionan sus necesidades de transporte con modos particulares, como las motos, el automóvil, la bicicleta y el transporte a pie. En cuanto al número de motos y vehículos registrados en la ciudad de Popayán, se tiene que, según datos de 
las Secretaría de Tránsito y Transporte, son 4214 motos y 10108 vehículos, pero hay que considerar, también, que algunas motos y vehículos que circulan en la ciudad están matriculados en Timbío, Cauca, por ser más baratos los impuestos en este municipio y por su cercanía. Otro transporte mencionado es el realizado en bicicleta, alternativa utilizada por una parte de la población. Aunque no hay datos sobre la demanda de este tipo de trasporte, el SETP contempla para la ciudad la adecuación y construcción de $15 \mathrm{~km}$ de ciclo rutas, frente a $88 \mathrm{~km}$ para el transporte colectivo. Este modo de transporte, que además de ser ecológicamente sostenible, representa una alternativa económica para la población de menores ingresos que no ha sido tenida muy en cuenta en los estudios de movilidad realizados en la ciudad. Finalmente se considera que el modo a pie es una alternativa a considerar, puesto que las distancias de los barrios periféricos al centro son relativamente cortas, y muchas personas optan por este tipo de desplazamiento.

\section{Modelación estocástica}

Si bien, desde un punto de vista teórico, el mejor modelo será aquel que mejor refleje el comportamiento real del individuo, en las aplicaciones reales debe adoptarse una decisión en cuanto al modelo a emplear, que puede no ser el teóricamente más adecuado. La principal limitación viene de que los modelos más complejos suelen precisar de una mayor calidad en los datos para poder ser estimados. Munizaga (1997), citado por Orro (2005), plantea que los modelos más sencillos utilizados actualmente se comportan bien en la mayoría de los casos, mientras propone que los modelos más generales solo sean utilizados en aquellos casos en los que el modelo logit simple presenta problemas. Para el caso particular de este trabajo se optó por un MNL, por dos razones: en primer lugar, la ciudad carece por completo de datos que permitan estudiar la elección modal, por tal razón, el estudio debe partir desde la recolección de información, y, en segundo lugar, con el cumplimiento de la independencia de alternativas irrelevantes, no se necesita acudir a la especificación de un modelo anidado, en un ejercicio previo se observó que evidentemente el MNL explicaba mejor la elección modal que el modelo NL.

\subsection{Modelo econométrico}

Para estudiar la demanda de transporte en la ciudad de Popayán, se optó por un modelo de elección modal, cuyas opciones son a pie, bicicleta, moto particular, mototaxi, colectivo, taxi, y automóvil particular. El ejercicio econométrico se enmarca en los modelos de elección discreta de respuesta múltiple para datos no ordenados, específicamente el modelo logit multinomial, pues el modo elegido se asocia con las características socioeconómicas de los individuos que toman la decisión. Ya que las variables ingresos y costos de transporte están muy relacionadas, se realizaron dos modelos, con el fin de evitar posibles problemas de multicolinealidad: territarias 33

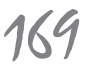


- Modelo 1:

modo $_{i}=\beta_{0}+\beta_{1}$ tiempo $_{i}+\beta_{2}$ genero $_{i}+\beta_{3}$ edad $_{i}+\beta_{4}$ jefeh $_{i}+\beta_{5}$ dingresol $_{\mathrm{i}}+$

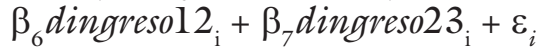

- $\quad$ Modelo 2:

$$
\text { modo }_{i}=\alpha_{0}+\alpha_{1} \text { tiempo }_{i}+\alpha_{2} \text { genero }_{i}+\alpha_{3} \text { edad }_{i}+\alpha_{4} j e f e h_{i}+\alpha_{5} \operatorname{costol}_{\mathrm{i}}+\pi_{i}
$$

El modo hace referencia a las siete posibles formas de transporte. Tiempo, son los minutos por desplazamiento desde el origen hasta el destino, género, es una variable dicotómica que toma el valor de 1 para hombre y 0 para mujer, edad, son los años del encuestado, jefeh, es una variable dicotómica que captura si es jefe de hogar o no ( si lo es, sume el valor de 1 y 0 en otro caso), dingresol, dingresol 2 y dingreso 23 son todas variables dicotómicas que muestran si los ingresos del individuo son menores o iguales a 1 salario mínimo, si están entre uno y dos, y si se ubican entre dos y tres, respectivamente. Finalmente, para el segundo modelo la variable costo muestra el costo total del desplazamiento. Las variables $\varepsilon_{i}$ y $\pi_{i}$ corresponden a los errores aleatorios, los cuales muestran las variables que influyen en la elección del modelo de transporte pero que no han sido tenidas en cuenta de manera explícita. Se asume que esta variable sigue una distribución logística.

A priori, se espera que en el caso de la variable tiempo, el signo sea negativo ya que, a medida que aumenta el tiempo por desplazamiento en los diferentes modos, existe más incentivo para utilizar el modo colectivo que es la categoría base. Para la variable género la expectativa es que guarde una relación positiva para la mayoría de los modos. Bajo la condición de ser hombre es posible que aumente la probabilidad de elegir modos más riesgosos o más rápidos que el colectivo. La variable edad puede presentar signos positivos para modos $\mathrm{co}^{-}$ mo caminar, taxi y automóvil particular, y signos negativos para las otras alternativas. Pues a medida que aumenta la edad se pueden preferir modos más seguros y saludables como caminar, taxi, o automóvil particular. Los signos asociados con la variable jefe de hogar pueden ser negativos para modos más costos (taxi y automóvil particular) y positivos para modos más económicos (a pie, bicicleta, moto, mototaxi), porque es de esperarse que el jefe de hogar trate de hacer algún tipo de economía en los gastos de transporte. Finalmente, frente a las variables de ingreso, se aguarda que los signos sean negativos para los modos más costos comparados con la categoría base, y positivos para los modos más económicos, también comparados con la categoría base. Es decir, para los individuos de ingresos bajos comparadas con los de ingresos altos, es más probable elegir modos más económicos que el colectivo, y menos probable elegir modos más costosos que el colectivo. 
Finalmente, con los costos se espera relaciones inversas para todas las categorías. Es decir, a mayores costos por modo aumenta la probabilidad de utilizar el transporte público colectivo.

\subsection{Minería de datos}

La ciudad de Popayán carece de una base de datos que permita estimar los modelos propuestos. Por tal razón, se procedió a implementar un muestreo aleatorio incidental a partir de una muestra, teniendo en cuenta el tamaño de la población del área urbana de la ciudad de Popayán en el año de estudio proyectada por el DANE. ${ }^{8} \mathrm{El}$ cálculo de la muestra para una población finita ascendió a 384 encuestas. En segundo lugar, las encuestas se realizaron con un muestreo casual o incidental en el centro de la ciudad y el barrio la Esmeralda, los dos lugares que mostraron según el SETP de la Alcaldía municipal (2008), la mayor afluencia de viajes según la matriz origen destino allí contenida. ${ }^{9}$

\subsection{Análisis descriptivo y resultados econométricos}

\subsubsection{Resultados generales de la encuesta}

La recolección de la base de datos concluyó con 389 encuestas validas, en donde el $53 \%$ de los individuos eran hombres y el $47 \%$ mujeres. Entre los encuestados, el modo de transporte más frecuente es el colectivo, con 118 observaciones, seguido por los modos a pie, con 73 ; moto particular, con 58; auto particular, con 49; mototaxi, con 39; bicicleta, con 32, y taxi con 20 observaciones. El rango de edad que más aparece en la muestra está entre los 21 y los 30 años. En cuanto al nivel de escolaridad, el 34,7\% de la muestra manifestó tener formación universitaria, el 29,8\% bachillerato, el 19,8\% formación técnica o tecnológica, seguidos de la formación primaria en un $7,7 \%$; solo el $7,2 \%$ de la muestra declaró tener formación a nivel de posgrado. Las principales actividades a las que se dedican los encuestados son trabajar y estudiar, con un $63,4 \%$ y un $23,1 \%$ respectivamente.

Además, la muestra arrojó un porcentaje de $52,2 \%$ de personas que manifestaron ser jefes de hogar, contra un $47,8 \%$ que no lo son. Frente a la variable ingresos, la encuesta arrojó que el 44,2\% de los encuestados tienen ingresos de un salario mínimo o menos, el 39,1\%, salarios mayores a un mínimo y menores que dos, el 10,8\%, salarios mayores a dos mínimos y menores que tres, y solo el 0,2\% manifestó tener ingresos mayores a tres salarios mínimos, tal como se aprecia en la figura 2.

En la variable motivo de viaje, se encontró que es el trabajo la actividad que más genera desplazamientos con 234 observaciones, seguida del motivo estudio con 82 observaciones (ver figura 3). Otros motivos que generan desplazamientos en la ciudad son el motivo asuntos personales, que registró 42 observaciones, seguido del motivo negocios, con 19 observaciones y, finalmente, el motivo compras, con 12 observaciones. De otro lado, teniendo en cuenta la relación entre el tiempo de viaje y
8 Siguiendo a Martínez (1998), la fórmula utilizada es la siguiente:

$n=\frac{N^{*} Z_{\alpha}^{2 *} p^{*} q}{d^{2}(N-1)+Z_{\alpha}^{2}{ }^{*} p^{*} q}$

Dónde N corresponde al total de la población, es el valor critico correspondiente al nivel de confianza elegido (95\%), p y q son las proporciones esperadas de un parámetro de la población $y d$ es el margen de error permitido. Para este caso en particular, se estableció un nivel de confianza del $5 \%$ y unas razones $p=0,5 y$ $q=0,5$ teniendo en cuenta la variable género, pues según datos del DANE, la población total de Popayán se compone, en un 48,3\%, de hombres, y un 51,6\% de mujeres.

${ }^{9}$ La ficha de la encuesta se relaciona en el anexo 1 .

territarias 33

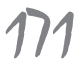


el modo de transporte, se encontró que el colectivo es el modo que utiliza más tiempo en promedio por trayecto, con $22,9 \mathrm{minu}^{-}$ tos, seguido del automóvil y la bicicleta, que utilizan 18,4 minutos en promedio; el modo a pie registra un tiempo promedio

Figura 2. Porcentaje por rangos de ingresos

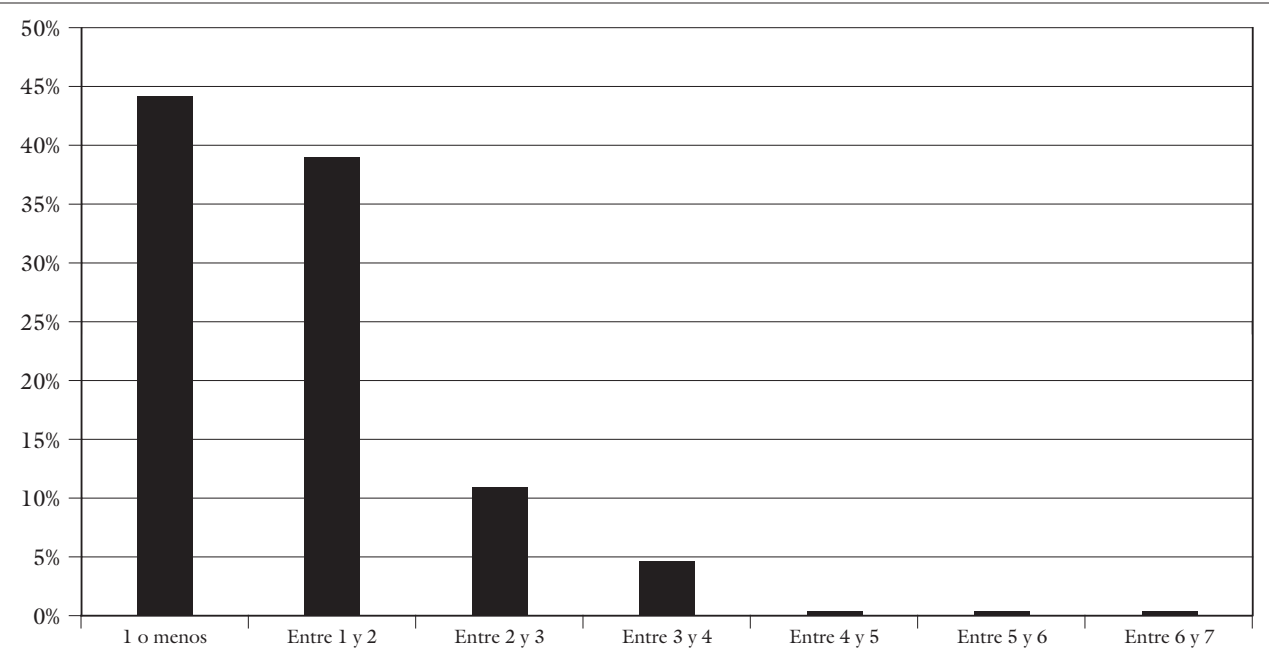

Fuente: elaboración propia.

Figura 3. Tiempo promedio de desplazamiento por modo 
de 17,4 min; le sigue el modo taxi con 14,5 min, la moto particular con 13,3 min y, finalmente, el modo más rápido es la mototaxi, con 11 min en promedio por trayecto.

\subsubsection{Estimación y Resultados Econométricos}

Como lo muestran los resultados presentados en la tabla 1 , la estimación del primer modelo arrojó un pseudo $\mathrm{R} 2=0,1579$, que como medida de bondad de ajuste es baja. ${ }^{10}$ De otro lado, es test de Hausman (no mostrado aquí) arrojó que se cumple la Independencia de Alternativas Irrelevantes (IAI), la cual establece que la probabilidad de elegir una alternativa determinada no se ve afectada por la presencia de alternativas adicionales.

De manera específica, la variable tiempo es significativa para los seis modos que se comparan con la categoría base (colectivo), el signo negativo es el esperado, pues tiene sentido que cuando el tiempo de desplazamiento aumenta por cada modo, esto incentiva el uso del transporte público

Tabla 1. Estimaciones del primer modelo de elección modal

\begin{tabular}{|l|c|c|c|c|c|c|c|c|c|c|c|c|}
\hline \multicolumn{7}{|c|}{ Regresión logitmultinomial } \\
\hline & \multicolumn{1}{|c|}{ A pie } & \multicolumn{1}{c|}{ Bicicleta } & \multicolumn{2}{c|}{ Moto } & \multicolumn{2}{c|}{ Moto-taxi } & \multicolumn{2}{c|}{ Taxi } & \multicolumn{2}{c|}{ Automóvil } \\
\hline VARIABLES & Coef. & Sig. & Coef. & Sig. & Coef. & Sig. & Coef. & Sig. & Coef. & Sig. & Coef. & Sig. \\
\hline Tiempo & $-0,069$ & $*$ & $-0,054$ & $*$ & $-0,131$ & $*$ & $-0,203$ & $*$ & $-0,128$ & $*$ & $-0,068$ & $*$ \\
\hline Género & 0,291 & & 0,111 & $*$ & 0,111 & $*$ & 0,055 & & $-0,650$ & & 0,498 & \\
\hline Edad & 0,039 & $*$ & 0,026 & & $-0,001$ & & 0,005 & & 0,055 & $*$ & 0,034 & $*$ \\
\hline Jefeh & 0,182 & & 0,117 & $*$ & 0,753 & $* *$ & 0,655 & & 0,441 & & $-0,266$ & \\
\hline Dingresol & 0,209 & & 0,203 & $*$ & 0,178 & $*$ & 0,124 & & $-0,197$ & $*$ & $-0,361$ & $*$ \\
\hline Dingresol2 & 0,241 & & 0,207 & $*$ & 0,181 & $*$ & $-0,118$ & & $-0,107$ & & $-0,223$ & $*$ \\
\hline Dingreso23 & 0,115 & & $-0,117$ & & 0,180 & $*$ & $-0,921$ & & 0,724 & & $-0,100$ & \\
\hline cons & $-0,869$ & & $-0,227$ & & $-0,173$ & & 0,275 & $*$ & $-0,215$ & & 0,135 & \\
\hline Categoría base: Colectivo & & & & & & & & & & & \\
\hline
\end{tabular}

Fuente: elaboración propia.

Categoría base: Colectivo. Significancia al $5 \%(*)$.

territarias 33

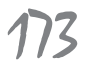


${ }^{11}$ Los resultados generales se presentan en el anexo 2.

${ }^{12}$ Los resultadosgeneralesse encuentran en el anexo 3.

\section{territarias 33} 174 colectivo y disminuye el uso de los otros modos. En el caso de la variable género, los signos son los esperados, salvo en el caso del taxi. Esta variable es significativa solo para los modos bicicleta y moto, lo cual representa que, dada la condición de ser hombre, aumenta la probabilidad de utilizar estos modos frente a la posibilidad de utilizar el servicio colectivo. La variable jefe de hogar presenta los signos esperados en todos los modos, menos en el modo taxi, y ella misma es significativa para los modos bicicleta y moto particular. Es decir que, bajo la condición de ser jefe de hogar, aumenta la probabilidad de utilizar estos modos en vez del colectivo. Los resultados resumidos se muestran a continuación. ${ }^{11}$

Las variables de ingresos muestran que en el caso del modo a pie, estas no son significativas, aunque tienen los signos esperados. Para el modo bicicleta, son significativas las variables de ingresos de uno y dos salarios mínimos, además de tener los signos esperados, lo que quiere decir que para los individuos de ingresos bajos aumenta la probabilidad de pasar del colectivo al modo bicicleta, comparados con los individuos de ingresos altos. Particularmente, se encontró que para el modo moto, todos los signos son los esperados y las variables de ingresos son también significativas, lo cual representa que para las personas cuyos ingresos están entre uno y dos salarios mínimos aumenta la probabilidad de utilizar las motos en vez del transporte colectivo, en comparación con los individuos que devengan más de tres salarios mínimos mensuales. Las variables de ingreso no son significativas en el caso del modo mototaxi.

Se encontró también que la variable ingresos de un salario mínimo o menos es significativa para el modo taxi y, además, tiene el signo esperado, lo cual significaría que, comparados los individuos de rentas bajas con los individuos de rentas altas, disminuye la probabilidad de los primeros de pasarse del modo colectivo al modo taxi. Y, finalmente, para el modo automóvil son significativas y con signos esperados las variables de ingresos de un salario mínimo y dos, pues, de la misma manera, se espera que la probabilidad de pasarse del modo colectivo al automóvil disminuya para estos individuos comparados con los de rentas altas.

El segundo modelo planteado en la metodología deja de lado las variables de ingresos e incluye la variable costo, conservando dentro de este las variables tiempo, género, edad y jefatura de hogar. Los resultados de este modelo se presentan en la tabla 2, donde el pseudo R2 es mayor que en el primer modelo e igual a 0,6203, indicando que la variable costo aporta de manera importante en el ajuste del modelo. Los resultados a manera de resumen se presentan en la tabla $2 .{ }^{12}$

En el anterior modelo, la variable género resulta significativa para el modo a pie y la variable jefe de hogar para el modo mototaxi, pero, aunque son significativas, no presentan los signos esperados. En el caso de la variable costos, se encontró que esta es significativa para todos los modos, menos para el modo a pie. Los signos son 
Tabla 2. Resultados del segundo modelo de elección modal

\begin{tabular}{|l|c|c|c|c|c|c|c|c|c|c|c|c|}
\hline \multicolumn{7}{|c|}{ Regresión logit multinomial } \\
\hline & \multicolumn{3}{|c|}{ A pie } & \multicolumn{2}{|c|}{ Bicicleta } & \multicolumn{2}{c|}{ Moto } & \multicolumn{2}{c|}{ Moto-taxi } & \multicolumn{2}{c|}{ Taxi } & \multicolumn{2}{c|}{ Automóvil } \\
\hline VARIABLES & Coef. & Sig. & Coef. & Sig. & Coef. & Sig. & Coef. & Sig. & Coef. & Sig. & Coef. & Sig. \\
\hline Tiempo & $-0,029$ & & $-0,005$ & & $-0,069$ & $*$ & $-0,291$ & $*$ & $-0,462$ & $*$ & $-0,392$ & $*$ \\
\hline Costo & $-0,341$ & & $-0,008$ & $*$ & $-0,004$ & $*$ & 0,002 & $*$ & 0,006 & $*$ & 0,006 & $*$ \\
\hline Género & $-0,154$ & $*$ & $-0,515$ & & 0,392 & & 0,585 & & $-0,052$ & & 0,131 & \\
\hline Edad & 0,027 & & 0,017 & & 0,003 & & $-0,013$ & & 0,018 & & 0,022 & \\
\hline Jefeh & 0,127 & & 0,112 & & 0,635 & & 0,114 & $*$ & 0,234 & & $-0,773$ & \\
\hline
\end{tabular}

Fuente: elaboración propia.

Categoría base: Colectivo. Significancia al $5 \%\left({ }^{*}\right)$.

los esperados en las categorías bicicleta y moto. Estos resultados indican que a mayores costos de estos modos, disminuye la probabilidad de usarlos en vez del modo colectivo. Los signos de los costos para las categorías mototaxi, taxi y automóvil, no son los esperados. La variable tiempo es significativa para los modos, moto, mototaxi, taxi y automóvil. Además, los signos son los esperados, pues se tiene que, a mayor tiempo necesario en cada modo, los individuos preferirán el modo colectivo. Estos resultados son los esperados, y son consistentes con otros ejercicios de este estilo, donde la aplicación del MNL llevó a concluir que el tiempo y los costos asociados con cada modo de transporte son variables importantes en la elección modal.

Finalmente, los efectos marginales encuentran que el transporte colectivo es el modo que mayor probabilidad promedio presenta de ser elegido con un $30 \%$, seguido del modo a pie con un valor de $18,7 \%$. Les siguen el modo moto y el modo automóvil con un $15 \%$ y un $13 \%$, respectivamente, de probabilidad promedio de ser elegidos. El modo mototaxi presenta una probabilidad promedio de elección del $10 \%$ $y$, finalmente, se encuentran el modo bicicleta y el modo taxi, con el $0,8 \%$ y el $0,5 \%$ de probabilidad promedio de elección respectivamente. De otro lado, las mujeres jefes de hogar presentan una probabilidad del $37 \%$ de elegir el modo colectivo, seguido del modo mototaxi con una probabilidad del $12 \%$; el modo que menor probabilidad tiene de ser elegido por las mujeres jefes de hogar es el modo bicicleta. En el caso de los hombres jefes de hogar, se encontró que el modo con mayor probabilidad de ser 
elegido es el colectivo, con $30 \%$, seguido de los modos a pie y automóvil, con una probabilidad del $28 \%$ y el $10 \%$, respectivamente. El modo que menor probabilidad tiene de ser elegido por los hombres jefes de hogar es el modo bicicleta, con un $2 \%$ de probabilidad. También se pudo estimar la probabilidad promedio de elección de cada modo, teniendo en cuenta las variables de ingreso, donde se encontraron las probabilidades que se explican a continuación.

Para los individuos que devengan un salario mínimo o menos, y para los que devengan entre uno y hasta dos, el modo con mayor probabilidad de ser elegido es la moto, con un $82 \%$; le sigue el modo bicicleta, con un $16 \%$, de la misma manera, los modos con menor probabilidad de ser escogidos para este grupo son el taxi, el automóvil y el mototaxi. Entre los individuos que devengan entre dos salarios mínimos y tres, el modo con mayor probabilidad de elección también es la moto, con un 99\% de probabilidad de elección. Otra relación interesante que se puede observar es la de la probabilidad de elección del modo y la edad; dicha relación se presenta en la figura 4.

La figura 5 muestra cómo para desplazamientos cuyo tiempo está entre 5 min y $10 \mathrm{~min}$ es mayor la probabilidad de elegir modos como a pie y mototaxi. El modo a pie tiene la mayor probabilidad de elección en desplazamientos de $15 \mathrm{~min}$; cuando los desplazamientos requieren un tiempo mayor de $30 \mathrm{~min}$, la probabilidad de elección baja considerablemente. El modo colectivo presenta las mayores probabilidades de elección en desplazamientos largos, es decir, cuando los desplazamientos tardan entre 55 y 60 minutos. Los modos moto, mototaxi y taxi, son los que tienen menor probabilidad de elección en la muestra cuando los desplazamientos son superiores a $25 \mathrm{~min}$. El automóvil no presenta cambios importantes en la probabilidad de elección en los desplazamientos de 5 min a 35 min; para desplazamientos que implican un mayor tiempo sí disminuye un poco la probabilidad de elección.

Se observa, claramente, cómo la población más joven tiene menor probabilidad de elegir el modo a pie y una mayor probabilidad de escoger el modo colectivo. A medida que aumenta la edad de los individuos encuestados, disminuye la probabilidad de elegir modos como la moto y el mototaxi, y disminuye más rápidamente la probabilidad de elegir el transporte colectivo. También se observa cómo la probabilidad de elegir el automóvil aumenta lentamente ante el incremento en la edad de los individuos encuestados. El modo taxi y el modo a pie presentan un incremento importante en la probabilidad de ser elegidos a medida de que los individuos de la muestra tienen más edad. Para terminar, se representa la relación entre la probabilidad de elegir los diferentes modos, en relación con el tiempo por desplazamiento.

\section{Algunos tópicos de discusión}

El plan de desarrollo 2006-2010 para Colombia llamó a su estrategia de desarrollo urbano "Ciudades Amables", donde el 
Figura 4. Probabilidad de elección de los modos por edad

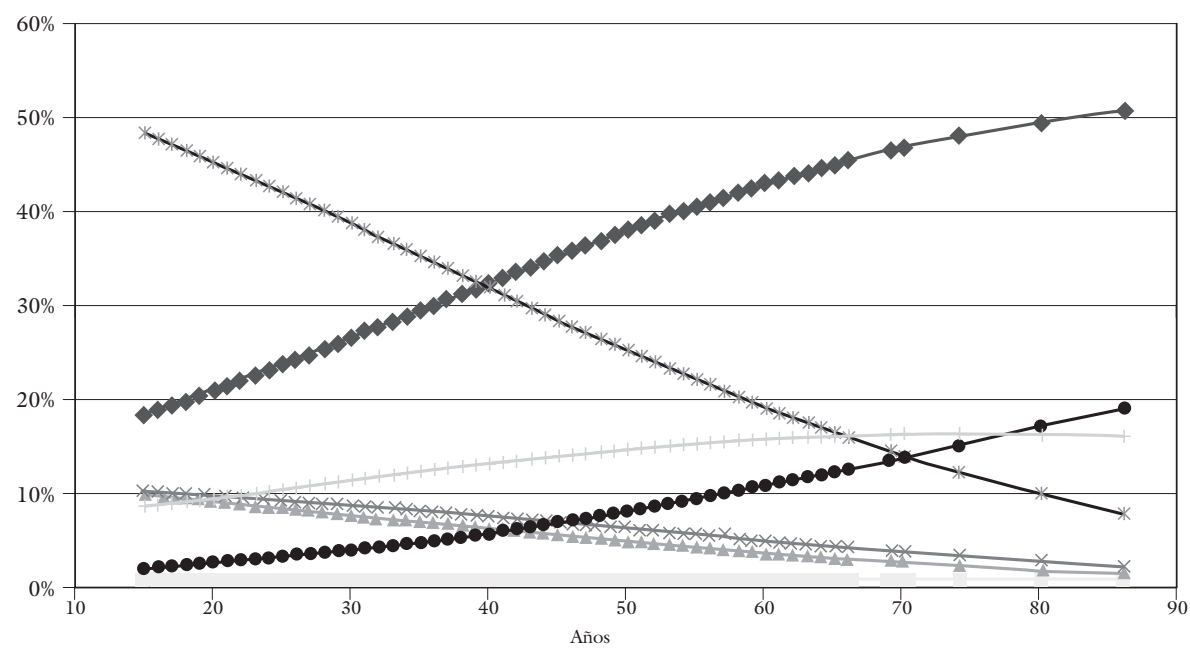

$$
\begin{aligned}
& \checkmark \text { prob A pie prob Bicicleta } \quad-1-\text { prob Moto } \\
& \leftarrow \text { prob Moto taxi } \rightarrow \text { prob Colectivo } \quad \longrightarrow \text { prob Taxi } \quad+\text { prob Automóvil }
\end{aligned}
$$

Fuente: elaboración propia.

Figura 5. Probabilidad de elección de los modos por tiempo

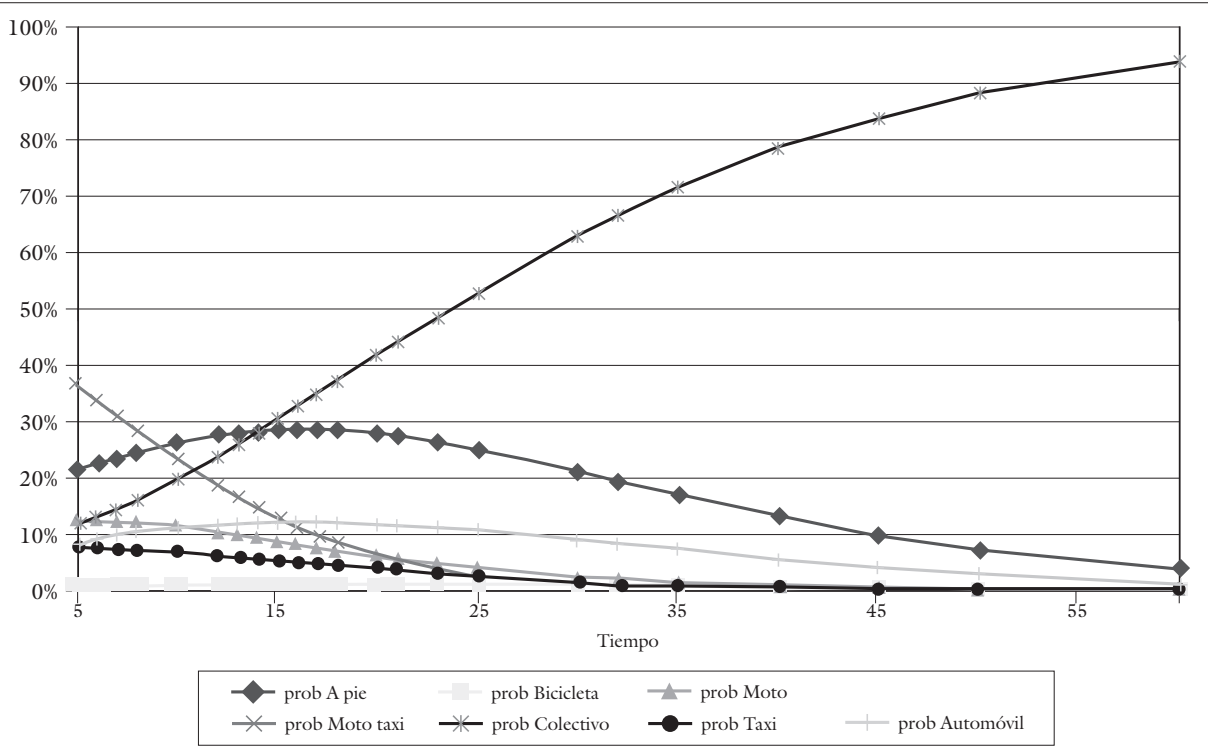

Fuente: elaboración propia. 
${ }^{13}$ Este concepto está asociado con quienes deben tener prioridad en el uso de la infraestructura para movilizarse en la ciudad, por su numero, por su eficiencia y vulnerabilidad. Este se representa en forma de pirámide invertida, en la que los peatones ocupan el lugar preferente, seguidos por los usuarios de la bicicleta, el transporte público, el transporte de carga y las motos $y$ autos particulares al final.

${ }^{14}$ Resolución del Parlamento Europeo del 12 de Octubre de 1988.

\section{territarias 33} 178 transporte y la movilidad eran uno de los ejes necesarios para lograr dicho desarrollo. La política de transporte, desde un principio, se dividió en dos grupos. El primero se aplicaba en ciudades con alta demanda, en las que se justificaba la intervención masiva de carriles exclusivos y de grandes inversiones en infraestructura, como es el caso de Transmilenio y sus similares, y el segundo era la promoción de un sistema estratégico de transporte público colectivo organizado (SET), que funcionaria sobre una misma plataforma tecnológica y que se aplicaría en ciudades intermedias de menos de 600000 habitantes. El objetivo era reorganizar y modernizar el transporte público colectivo en doce ciudades en las cuales se incluía Popayán. Dichos cambios comprendían no solo cambios empresariales en la estructura (que todavía permanecía con el esquema de afiliadores, con la sobreoferta y la guerra del centavo) sino que también incluía la incorporación de un sistema de recaudo unificado (de pago con tarjetas) y otro de control de información de tránsito y transporte.

En la ciudad de Popayán, el ente gestor del proyecto era el Sistema Estratégico de Transporte Público Colectivo SETPC, que es la empresa Movilidad Futura S.A.S. La primera fase del proyecto estaba pensada como un sistema de transporte público y no como un plan de movilidad y, solo hasta 2014 , se empezó a pensar en la necesidad de solucionar el problema de movilidad de manera integral con propuestas más inclusivas que consideraran la jerarquía de la movilidad y los demás modos de transpor- te. ${ }^{13}$ En este orden de ideas, las inversiones en infraestructura dan cuenta de cuál es el modo de transporte que se prioriza.

El Sistema Estratégico de Transporte Público de Popayán contempla dentro de sus obras la construcción de puentes peatonales como la solución principal para la protección del peatón y la reducción de la accidental, sin embargo, la empresa enfatiza en que los peatones son reacios a utilizar estas estructuras. Cuando se le obliga al peatón a gastar más tiempo en sus desplazamientos por la utilización de los puentes se le está dando un lugar abajo en la jerarquía de la movilidad. La infraestructura vial debe considerar la movilidad segura de los peatones, más allá de los puentes elevados, pensando también en incluir alternativas como los cruces tipo cebra con prioridad al peatón, corredores integrados con reductores de velocidad, cruces semafóricos, entre otras alternativas. Esta es una preocupación de muchas naciones, es así como la Unión Europea y la Comunidad Latinoamericana de Naciones ha trabajado en identificar y normalizar los derechos de los peatones. ${ }^{14}$

En el caso del modo bicicleta, el SETP plantea la construcción de $19 \mathrm{Km}$ de ciclorutas, que todavía no se han construido. En el proyecto se planteaba una cicloruta que permitiera a los usuarios de las bicicletas trasladarse de manera segura del norte al centro de la ciudad y del oriente al centro, conectando las zonas de mayor actividad económica de la ciudad como el centro y La Esmeralda, con los barrios más alejados y de menores estratos económicos de la ciudad. Es de esperarse que una alternati- 
va como esta redunde directamente en el bienestar de la población, pues el transporte tiene un peso importante en los gastos del hogar.

El problema de la movilidad en la ciudad de Popayán se ve reforzado por la presencia del mototaxi, que, como modo de transporte, es inseguro e ilegal, pero que tiene una fuerte ventaja frente a la opción de transporte público colectivo y es el tiempo por desplazamiento, además, la elección de la moto como medio de transporte puede ser una alternativa racional en el mejor sentido económico, pues no solo soluciona el problema de en qué movilizarse a muy bajo costo, sino que también puede ser una fuente de ingresos, si se utiliza para prestar el servicio de transporte. Este modo de transporte comparte la infraestructura con el transporte público y los vehículos particulares, sumado a esto la malla vial se encuentra en reparación y construcción, lo que genera mayores problemas de movilidad.

El problema del transporte ilegal e informal se explica no solo por la existen-

Figura 6. Red de Ciclo Rutas propuesta en el SETP de Popayán

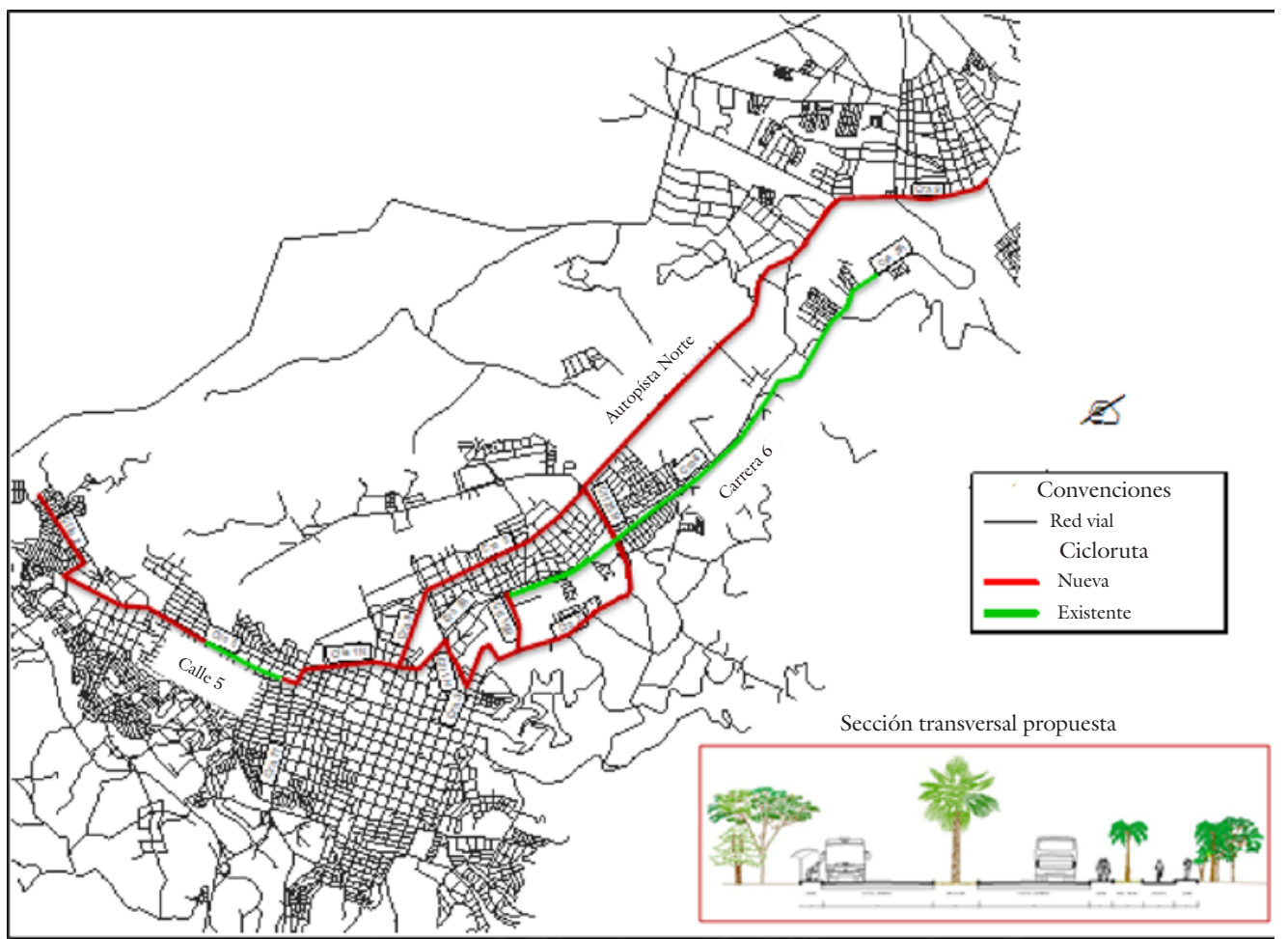

Fuente: diseño conceptual del sistema estratégico de transporte público de Popayán. 
cia de un sistema de transporte público ineficiente sino también por la estructura económica de la ciudad, donde el desempleo alcanza el 19\% en los últimos cinco años, el 95,5\% de las empresas registradas en 2012 eran microempresas y el 62,1\% de los ocupados estaban el categoría cuenta propia (ORMET Popayán, 2013), por lo que una alternativa de colocación es el mototaxismo. Sumado a esto, las soluciones hasta ahora planteadas por la alcaldía municipal, como el pico y placa o la prohibición del parrillero en el centro de la ciudad, no atacan la principal ventaja de este modo de transporte: el tiempo por desplazamiento. Es decir, mientras el desplazamiento en mototaxi le represente menor tiempo de desplazamiento al usuario y los precios del servicio sean similares a los del transporte legal, sin importar la carencia de seguridad del transporte ilegal, su elección se inclinará en la mayoría de los casos a dicho modo de transporte.

De otro lado, la figura 7 muestra que los desplazamientos de los payaneses en los diferentes modos de transporte provienen en su mayoría del norte y sur occidente de la ciudad y su destino es la zona centro y la zona de la galería La Esmeralda, la cual es la plaza de mercado más grande de la ciudad. Esto indica que la capital caucana es todavía una ciudad centralizada donde la alcaldía, gobernación, entidades bancarias, comercio, iglesias, colegios, clínicas y hospitales, entre otros, se encuentran ubicadas en esta parte de la ciudad, lo que influye de manera directa en el problema del transporte.
En efecto, la implementación de un modelo de ciudad que intente resolver los problemas de movilidad debe pasar por descentralizar la ciudad para que transportes no contaminantes como las bicicletas no deban recorrer grandes distancias, el centro pueda ser peatonalizado totalmente y la congestión, la contaminación, al igual que la accidentalidad, disminuyan. Adicionalmente, los recorridos serían más cortos, lo que sería valorado por los usuarios del transporte público y, posiblemente, cambiarían sus hábitos de demanda por transporte ilegal.

Cabe decir, finalmente, que en los últimos años y, gracias a la inversión en el mejoramiento del Sistema de Transporte Público de la ciudad, el problema de la movilidad es un tema obligado para los gestores de política pública. Se ha avanzado en el cambio del sistema de cobro de las carreras en taxi, se construye actualmente el plan de movilidad y se realizan las obras con una visión de conservación del patrimonio, pero aún queda mucho por resolver, no solo en inversiones sino también en cultura ciudadana.

\section{Comentarios finales y recomendaciones}

De acuerdo con los resultados anteriores, se puede decir que los costos, el tiempo, los ingresos y la edad, son determinantes en la elección modal en la ciudad de Popayán, a diferencia de variables como el género y el ser o no jefe de hogar. Este resultado es 


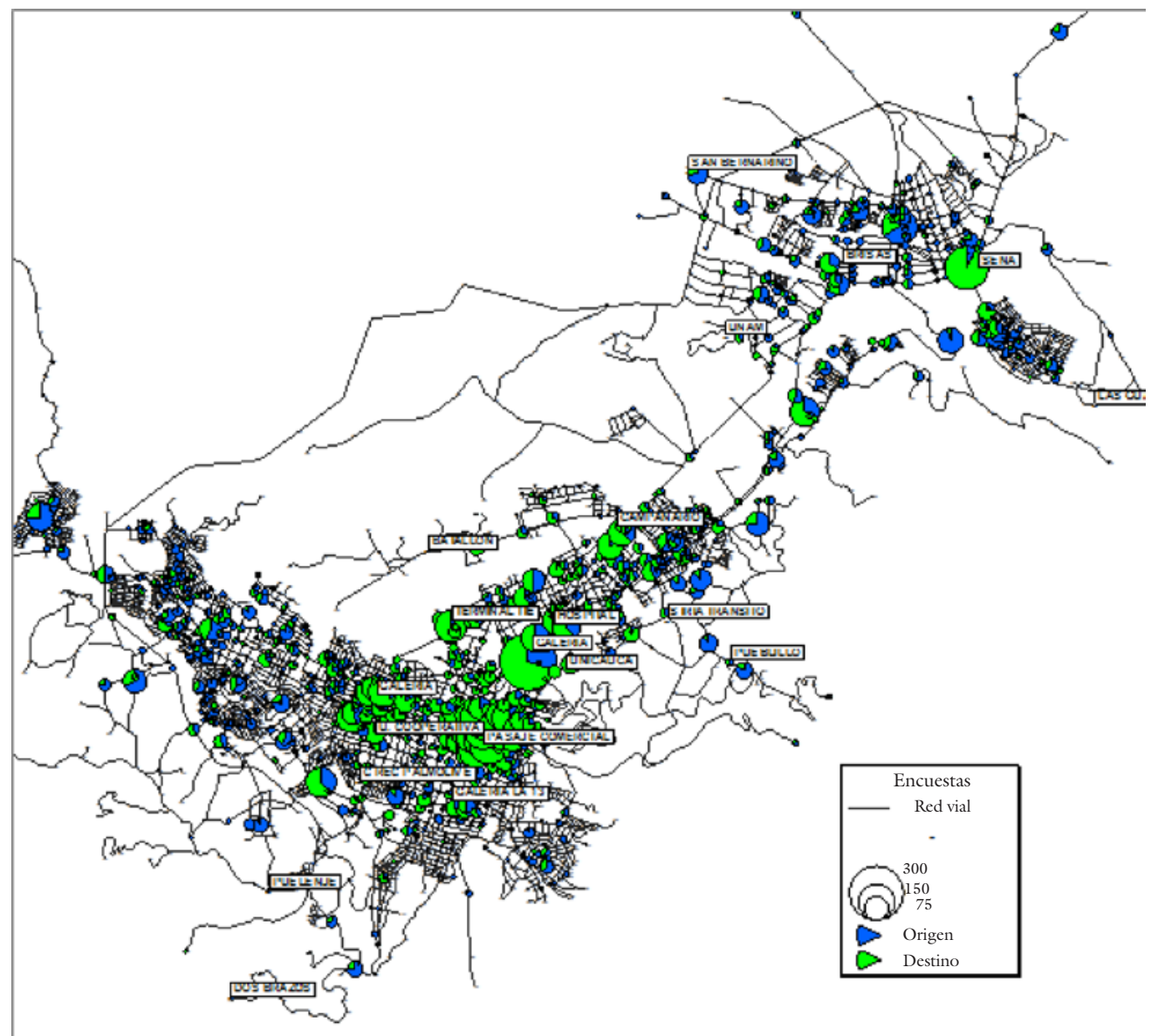

Fuente: Alcaldía de Popayán, SETP Informe 3.

consecuente con los hallazgos de ejercicios similares en otras partes del país y del mundo. La variable costos mostró ser significativa en las categorías bicicleta, moto, mototaxi, taxi y automóvil y, aunque los signos no fueron los esperados en estas últimas dos categorías, hay varias reflexiones al respecto de este resultado. En primer lugar, aquellas decisiones que afecten los costos de modos como el taxi y el automóvil pueden no influir en la elección de este modo, por tal razón, el control del uso del vehículo, para evitar congestiones, por la vía de los costos puede no tener mucho éxito. En segundo lugar, la elección del modo de automóvil particular es elevada, si se compara con la territarias 33 
población que tiene ingresos superiores a tres salarios mínimos, siendo así, la decisión de tener vehículo particular como modo de transporte puede deberse a otros factores diferentes a los costos por desplazamiento, en esta alternativa.

A través de los dos modelos se encuentra que el factor tiempo es clave en la elección modal. Esto es de gran importancia en el manejo de la política de transporte público, pues el sistema de transporte público informal (mototaxi) tiene esta ventaja sobre los demás modos en este sentido, ya que presenta los menores tiempos promedio por desplazamiento. Este resultado permite pensar que las medidas de control del transporte público informal, que no impliquen una disminución de los tiempos por desplazamiento de los modos que compiten con este servicio, no tengan resultados favorables. Del lado de los ingresos de los individuos de rentas bajas, comparados con los de rentas altas, explican la elección de modos como la moto, la bicicleta, el taxi y el automóvil. Modos como a pie y mototaxi son explicados por otras variables. Se encontró, así mismo, que los modos que tienen mayor probabilidad de ser elegidos por las personas de rentas bajas son la moto, en primer lugar, $y$, posteriormente, la bicicleta, lo cual trae consigo una importante implicación en términos de políticas públicas, debido a que las decisiones que restringen el uso de este medio perjudican a una importante fracción de la población. Ejemplo de lo anterior es la medida del pico y placa para las motos en toda la ciudad, que rige hace ya algún tiempo. Los impac- tos de esta decisión de política pueden ser objeto de estudio en posteriores trabajos.

Los resultados encontrados permiten aventurarse en algunas recomendaciones de política económica en materia de transporte público. En primer lugar, y teniendo en cuenta que modos más económicos son ampliamente elegidos por la población, políticas orientadas a incentivar el uso del modo a pie, bicicleta y moto particular, pueden impactar significativamente en la población, es decir, mejores condiciones de seguridad en las vías, ciclo rutas y cultura ciudadana que permita hacer uso de estos modos de forma más segura y generalizada, pueden ser alternativas para mejorar la movilidad en la ciudad.

La construcción de la ciclo ruta estipulada en el proyecto de SETP permitirá a población de menores ingresos mejorar sus condiciones de vida en la medida que brindará la posibilidad de desplazarse a los dos principales centros de actividad económica de la ciudad de manera segura y a bajo costo. Esto, sin contar con el impacto positivo sobre la población que utiliza bicicleta con fines deportivos. Empero, mientras la centralización de la ciudad sea tan alta, es poco probable que esto suceda. Se requiere un diseño de ciudad diferente, como el de algunas ciudades europeas, donde las distancias recorridas sean cortas y para llegar a las más distantes se cuente con un sistema de desplazamiento (metros o buses articulados) que permitan ingresar las bicicletas a él o, en su defecto, existan bicicletas públicas en las zonas de destino. 
De otro lado, si se quiere incentivar el uso del transporte público colectivo, es necesario tener en cuenta tanto el tiempo como el costo por desplazamiento. Por un lado, el aumento en términos reales de la tarifa en los últimos años hace que el desplazamiento en colectivo se haga caro, comparado con modos como la moto particular, cuyo costo se estimó en aproximadamente de \$ 165 por km (para una moto de cuatro tiempos y de 110 cilindros). Así mismo, el colectivo compite con los mototaxis, que tienen los menores tiempos por desplazamiento de todos los modos. Este último medio de transporte, aunque inseguro, ha subsistido en el mercado, a pesar de las medidas tomadas por la alcaldía municipal (retenes, multas, pico y placa y restricción de parrilleros en el centro de la ciudad), toda vez que no se ha atacado su principal ventaja competitiva: el tiempo. Entonces, las recomendaciones para mejorar el uso del transporte público colectivo son mantener el valor real de la tarifa y disminuir los tiempos promedio por desplazamiento.

Parte de las políticas de transporte público en las ciudades busca disminuir el uso de automóviles particulares, y así disminuir la presión sobre la infraestructura existente, pues nuevas carreteras requieren de tiempo y grandes inversiones. El problema con este modo en la ciudad es que la elección de este parece no estar explicada ni por los costos, ni por los ingresos, por ende, encarecer su uso puede no ser la política más adecuada. Por consiguiente, se hace necesario indagar aún más sobre las razones por las cuales los payaneses compran vehículo. La política pública debe empezar por reconocer la jerarquía de la movilidad, si no, las soluciones para los modos más eficientes de transporte seguirán siendo las equivocadas, como es el caso de los puentes peatonales que aumentan los tiempos por desplazamiento de los peatones y priorizan el modo menos eficiente, como lo es el automóvil particular. Brindar a la población la posibilidad de elegir modos de desplazamiento más económicos y eficientes es una alternativa que no solo ayuda a mejorar la movilidad sino también el bienestar de las familias con menores ingresos.

Para finalizar, cabe hacer algunas consideraciones sobre algunos resultados y posibles líneas de trabajo en el área de la demanda de transporte para la ciudad a futuro. En primera instancia, la variable género puede tener más peso explicativo si la elección modal tiene que ver con el motivo por el cual los ciudadanos viajan, es decir, los ejercicios de elección modal que implican el motivo del viaje encuentran en la variable género una variable explicativa de mayor participación. Así mismo, el estudio debería abordar un análisis detallado del impacto del ingreso en la elección modal, compaginando los resultados obtenidos con información sobre la línea de pobreza de la ciudad y por rangos sociodemográficos.

Una línea de trabajo posible a seguir es el análisis de las políticas públicas vigentes en la ciudad. Este ejercicio podría realizarse desde un enfoque que involucre tanto los costos privados como los costos sociales de algunas de ellas, por ejemplo, las motos particulares que, si bien es cierto territarias 33

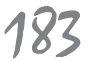


tienen bajos costos privados, pueden tener altos costos sociales en términos de contaminación y accidentalidad, motivar su uso puede no ser una solución viable a largo plazo. Este caso particular y la discusión sobre la viabilidad del sistema integrado de transporte en la ciudad son temas totalmente pertinentes, dado la coyuntura que atraviesa no solo la ciudad sino también el país en materia de transporte público.

\section{Referencias}

Alcaldía de Popayán (2008). Sistema Estratégico de Transporte Público Colectivo, SETP. Recuperado de http:// movilidad-popayan-cauca.gov.co/ apc-aafiles / 63646462633262376 635316530633063/convenio_de_cofinanciacion0001.pdf

Alcaldía de Popayán (2009). Diseño conceptual del Sistema Estratégico de Transporte Público de Popayán. Contrato de servicios de consultoría N. ${ }^{\circ}$ sub0000003099. Informe ejecutivo.

Alvis, J., Arellano, W., \& Toro, D. (2005). Transporte público en Cartagena: ¿Qué factores determinan las preferencias de los usuarios? Economia y Región, 2(3), 7-54.

Causado, E., De la Hoz, B., \& Cantillo, L. (2009). Dinámica del transporte público terrestre automotor en la ciudad de Santa Marta 2005-2006. Clío América, $3(6), 257-281$.

Chavarriaga, F. (2006). Análisis de decisión modal y bienestar: Una estimación de la demanda de transporte basado en la en- cuesta de movilidad. Memorias de grado, Facultad de Economía, Universidad de los Andes, Bogotá, Colombia.

De Rus, G., Campos, J., \& Nombela, G. (2004). Economia del transporte. Gran Canaria: Antoni Bosh Editores.

Galán, J. (2005). Determinantes de la Demanda por Transporte Público y Privado en el Área Metropolitana de Monterrey. Ciencia UANL, 8(4), 495-501.

González, R. (1995). La demanda de transporte de pasajeros en el trayecto Gran Canaria-Tenerife. Una aplicación de los modelos de elección discreta. (Tesis doctoral, Universidad de la Laguna, Tenerife).

Greene, W. (1999). Econometric Analysis. 5. ${ }^{a}$ ed. New Jersey: Prentince Hall.

ICER (2012). Informe de Coyuntura Económica Regional. Cauca. Semestre II DANE.

MacFadden, D. (1974). The Measurement of Urban Travel Demand. Journal of Public Economics, 3, 303-328.

Martín, J., \& Román, C. (1999). Análisis de la Demanda de Transporte en España. Papeles de Economía Española, 82, 88-106.

Martínez, C. (1998). Estadística y muestreo. Bogotá: Ecoe Editores.

Moreno, D., Sarmiento, I., \& González-Calderón, C. (2010). Políticas para influir en la elección modal del uso de vehículos privados en la universidad: El caso de la Universidad de Antioquia, Dyna, 78(165), 101-111.

Medina, E. (2003). Modelos de Elección Discreta. Universidad Autónoma de Madrid. Working Paper, 26 p. 
Mendieta, J., \& Perdomo, A. (2008). Fundamentos de economía del transporte: Teoría, metodología y análisis de politica, Bogotá: Editorial Uniandes.

Munizaga, M. A. (1997). Implicancias de la naturaleza de los datos en la modelación de elecciones discretas. (Tesis Doctoral, Pontificia Universidad Católica de Chile, Escuela de Ingeniería, Chile).

ormet (2013). Diagnóstico del Mercado Laboral de Popayán. Popayán: Observatorio Regional del Mercado de Trabajo. Ministerio de Trabajo, Departamento de la Protección Social y Plan de Naciones Unidas para el Desarrollo.

Orro, A. (2005). Modelos de elección discreta en transportes con coeficientes aleatorios. (Tesis Doctoral, Escuela Técnica Superior de Ingenieros de Caminos,
Canales y Puertos. Universidad de la Coruña, España).

Roa, D. (2009). Análisis de los factores que han limitado la implementación de los Decretos 112 a 116 de 2003 de reestructuración del sistema de transporte público colectivo en Bogotá. (Tesis, Universidad Colegio Mayor de Nuestra Señora del Rosario, Bogotá).

Rodrigue, J. P. (2013). The Geography of Transport Systems, 3. ${ }^{a}$, New York: Routledge.

Train, K. (2003). Discrete choice analysis methods with simulation. Cambridge: Cambridge University Press.

Vivas, H. (2009). Notas de clase: curso de Economía del Transporte. Maestría en Economía Aplicada. Universidad del Valle. 


\section{Anexos}

\section{Anexo 1. Encuesta}

Información general sobre el modo de transporte.

A. Hacia dónde se dirige:

Motivo de viaje:
(1) Trabajo
(2) Estudio
(3) Compras
(4) Diligencias personales
(5) Ocio
(6) Otra: ¿Cuál?

B. Señale cuál es el medio de transporte que normalmente utiliza para acudir a sus actividades diarias (Solo uno)

Modo:
(1) A pie
(2) Bicicleta
(3) Moto particular o propia
(4) Mototaxi
(5) Colectivo
(6) Taxi
(7) Automóvil particular

C. ¿Cuál es la principal razón por la que elige este medio de transporte? Características del modo:
(1) Seguridad
(2) Rapidez
(3) Disponibilidad
(4) Comodidad
(5) Precio
(6) Salud
(7) Cercanía a su destino más frecuente. 
D. ¿Con qué fin se moviliza principalmente en la ciudad! Motivo de viaje:
(1) Trabajo
(2) Estudio
(3) Negocios
(4) Compras
(5)Asuntos personales

E. ¿Cuánto tiempo en promedio (minutos) dura su desplazamiento más frecuente desde su origen hasta su destino?

Tiempo:

Minutos:

F. ¿Cuánto paga por su desplazamiento más frecuente?

Costo: $\$$

Parqueadero por hora: $\$$

(para vehículos propios)

G. ¿Cuál es la distancia aproximada de su trayecto más frecuente o cuál es el origen de su desplazamiento más frecuente y su destino?

Distancia:

$\mathrm{Km}$

Metros

Cuadras

Origen

Destino

Información general del encuestado
A. Género:
Hombre

Mujer (_

B. Edad:

Años

C. ¿Cuál es su nivel de escolaridad!

Educación:

(1) Ninguno

(2) Preescolar y primaria 

(3) Bachillerato
(4) Técnico /Tecnológico
(5) Universitario
(6) Posgrado

D. ¿¿Cuál es su actividad principal? ¿A qué se dedica principalmente? Actividad principal:
(1) Estudiar
(2) Trabajar
(3) Oficios del hogar
(4) Jubilado
(5) Rentista
(6) Busca trabajo
(7) Incapacitado permanentemente para trabajar

E. ¿Es usted jefe de hogar?

Jefe del Hogar:

Sí

No

F. ¿A cuánto ascienden sus gastos personales al mes?

Valor aproximado $\$$

(1) Menos de un salario mínimo

(2) Entre un mínimo y dos (\$ 589500 - \$ 1178000$)$

(3) Entre dos mínimos y tres (\$ 1178.000 - \$ 1767000)

(4) Entre tres mínimos y cuatro. (\$ 1767000 - \$356000)

(5) Entre cuatro mínimos y cinco (\$2356000 - \$2945000)

(6) Entre cinco mínimos y seis (\$2945000 - \$3534000)

(7) Entre seis mínimos y siete (\$ 3534000 - 4123000)

(8) Entre siete mínimos y ocho (\$ 4123000 - \$ 4712000)

(9) Entre ocho mínimos y nueve (\$ 4712000 - \$5301000)

(10) Entre nueve mínimos y diez (\$5301000 - \$5890000)

(11) Más de diez mínimos (> \$5890000) 


\section{Anexo 2. Estimaciones Modelo 1}

. mogit modo ti empo gnero edad jefeh dingresol dingresol2 dingreso23, nol og Mil ti nomial logistic regression Log 1 i kel i hood $=-592.69029$ Number of obs $=389$ $\begin{array}{lll}\mathrm{LR} c h i 2(42) & = & 222.19\end{array}$ $\begin{array}{lll}\text { Proudo R2 } & = & 0.0000 \\ & & 0.1579\end{array}$

\begin{tabular}{|c|c|c|c|c|c|c|}
\hline modo & Coef. & St d. Er $\mathrm{r}$. & $\mathrm{z}$ & $P>|z|$ & {$\left[\begin{array}{lll}95 \% & \text { Conf }\end{array}\right.$} & Interval ] \\
\hline \multicolumn{7}{|l|}{1} \\
\hline t i e npo & -.0689928 & 017658 & -3.91 & 0.000 & - . 1036019 & 0343837 \\
\hline gnero & 291157 & 3157012 & 0.92 & 0.356 & -.327606 & 9099201 \\
\hline edad & .0390893 & 0149581 & 2.61 & 0.009 & 009772 & 0684065 \\
\hline jefeh & .1821827 & 3844264 & 0.47 & 0.636 & -.5712792 & 9356446 \\
\hline di ngresol & 2086806 & 9367002 & 0.22 & 0.824 & -1.627218 & 2. 044579 \\
\hline di ngresol2 & 2414613 & 9354212 & 0.26 & 0.796 & -1.591931 & 2. 074853 \\
\hline dingreso23 & 1. 148191 & 1. 04798 & 1. 10 & 0.273 & -.9058114 & 3. 202193 \\
\hline _cons & -.8688061 & 1. 084553 & -0.80 & 0.423 & -2.99449 & 1. 256878 \\
\hline \multicolumn{7}{|l|}{2} \\
\hline t i enpo & -0541592 & 0219283 & -2.47 & 0.014 & -.097138 & -.0111804 \\
\hline gnero & 1. 114863 & 4517437 & 2.47 & 0.014 & .2294615 & 2. 000264 \\
\hline edad & .0257621 & 0185819 & 1. 39 & 0.166 & -0106576 & .0621819 \\
\hline jefeh & 1. 171233 & 5338363 & 2.19 & 0.028 & 124933 & 2. 217533 \\
\hline di ngresol & 20.34369 & 7136524 & 28.51 & 0.000 & 18.94496 & 21.74243 \\
\hline di ngres ol 2 & 20.66497 & 8076945 & 25.59 & 0.000 & 19.08191 & 22. 24802 \\
\hline di ngreso23 & -11.74725 & 6572202 & -0.00 & 1.000 & $-1.29 \mathrm{e}+07$ & 1. $29 \mathrm{e}+07$ \\
\hline _cons & -22.71877 & & & & 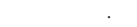 & \\
\hline \multicolumn{7}{|l|}{3} \\
\hline t i empo & -.1313698 & 0246367 & -5.33 & 0.000 & - . 1796567 & -.0830828 \\
\hline gnero & 1. 109589 & 3703203 & 3.00 & 0.003 & 383774 & 1. 835403 \\
\hline edad & -0005652 & 0192623 & -0.03 & 0.977 & -0383187 & 0371883 \\
\hline jefeh & .7534464 & 4327723 & 1.74 & 0.082 & . . 0947718 & 1. 601665 \\
\hline di ngresol & 17.80776 & 6681965 & 26.65 & 0.000 & 16.49812 & 19. 1174 \\
\hline di ngresol2 & 18.08291 & 7452202 & 24.27 & 0.000 & 16.6223 & 19. 54351 \\
\hline di ngreso23 & 17.98299 & 1. 062093 & 16.93 & 0.000 & 15.90132 & 20.06465 \\
\hline _cons & -17.29838 & 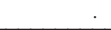 & & & & \\
\hline \multicolumn{7}{|l|}{4} \\
\hline t i e mpo & - . 2029079 & 0333497 & -6.08 & 0.000 & -.2682722 & -.1375436 \\
\hline gnero & .0546698 & 4064935 & 0.13 & 0.893 & -.7420428 & 8513824 \\
\hline edad & 0048217 & 0217756 & 0.22 & 0.825 & -.0378577 & .047501 \\
\hline jefeh & 6550904 & .493599 & 1. 33 & 0.184 & -.312346 & 1. 622527 \\
\hline di ngresol & -1.237704 & 9497919 & -1.30 & 0.193 & -3.099261 & 6238544 \\
\hline di ngresol 2 & -1.17754 & 9300031 & -1.27 & 0.205 & -3.000312 & 6452327 \\
\hline di ngreso23 & -.9213488 & 1. 209292 & -0.76 & 0.446 & -3.291517 & 1. 448819 \\
\hline _cons & 2. 74942 & 1. 254076 & 2. 19 & 0.028 & .2914756 & 5. 207365 \\
\hline \multicolumn{7}{|c|}{1057205} \\
\hline t i enpo & 12837 & 0343682 & -3.74 & 0.000 & -1957305 & -0610095 \\
\hline gnero & -.6501904 & 5467832 & -1.19 & 0.234 & -1.721866 & 4214849 \\
\hline edad & .0545574 & .021936 & 2. 49 & 0.013 & .0115636 & 0975512 \\
\hline jefeh & 440557 & 629711 & 0.70 & 0.484 & -.7936539 & 1. 674768 \\
\hline di ngres ol & -1.965274 & 1. 096951 & -1.79 & 0.073 & -4.115259 & .184711 \\
\hline di ngresol2 & -1.073564 & 1. 019773 & -1.05 & 0.292 & -3.072282 & .925155 \\
\hline di ngreso23 & .7240977 & 1. 110719 & 0.65 & 0.514 & -1.452872 & 2. 901068 \\
\hline _cons & -.2153908 & 1. 384152 & -0.16 & 0.876 & -2.928278 & 2. 497497 \\
\hline \multicolumn{7}{|c|}{. . 1108243} \\
\hline t i enpo & -.0678639 & 021919 & -3.10 & 0.002 & -1108243 & - . 0249035 \\
\hline gnero & 4981012 & 4016594 & 1. 24 & 0.215 & -.2891368 & 1. 285339 \\
\hline edad & 0337918 & 019068 & 1.77 & 0.076 & .0035808 & 0711645 \\
\hline jefeh & -.2662332 & 4671566 & -0.57 & 0.569 & -1.181843 & .649377 \\
\hline di ngresol & -3.608617 & 7865295 & -4.59 & 0.000 & -5.150186 & -2.067047 \\
\hline di ngresol2 & -2.226388 & 7021065 & -3.17 & 0.002 & -3.602491 & -.8502843 \\
\hline di ngreso23 & -.1002845 & 7925934 & -0.13 & 0.899 & -1.653739 & 1. 45317 \\
\hline _cons & 1. 349516 & 1. 032358 & 1. 31 & 0.191 & -.6738674 & 3.3729 \\
\hline
\end{tabular}

( $\operatorname{modo}==5$ is the base out come) 
Anexo 3. Estimaciones Modelo 2

m ogit modo ti empo costo gnero edad jefeh, nol og

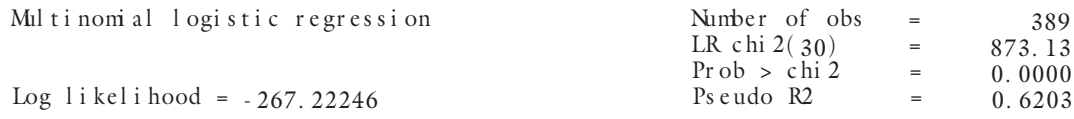

\begin{tabular}{|c|c|c|c|c|c|c|}
\hline modo & Coef. & St d. Er r. & $\mathrm{z}$ & $\mathrm{P}>|\mathrm{z}|$ & {$\left[\begin{array}{lll}95 \% & \text { Conf }\end{array}\right.$} & Int erval ] \\
\hline $\begin{array}{r}\text { t i e mpo } \\
\text { costo } \\
\text { gnero } \\
\text { edad } \\
\text { jefeh } \\
\text { _cons }\end{array}$ & $\begin{array}{r}-.0287628 \\
-34.12728 \\
-1.543577 \\
.0268035 \\
.1273819 \\
5.954247\end{array}$ & $\begin{array}{l}.0419432 \\
8420713 \\
.8228057 \\
.0312903 \\
.8180123 \\
1.342397\end{array}$ & $\begin{array}{r}-0.69 \\
-0.00 \\
-1.88 \\
0.86 \\
0.16 \\
4.44\end{array}$ & $\begin{array}{l}0.493 \\
1.000 \\
0.061 \\
0.392 \\
0.876 \\
0.000\end{array}$ & $\begin{array}{r}-.1109701 \\
-1.65 \mathrm{e}+07 \\
-3.156246 \\
-.0345243 \\
-1.475893 \\
3.323197\end{array}$ & $\begin{array}{l}.0534444 \\
1.65 \mathrm{e}+07 \\
.0690925 \\
.0881313 \\
1.730657 \\
8.585297\end{array}$ \\
\hline $\begin{array}{r}\text { t i e mpo } \\
\text { costo } \\
\text { gnero } \\
\text { edad } \\
\text { jefeh } \\
\text { _cons }\end{array}$ & $\begin{array}{r}-.005412 \\
-.0076477 \\
-.5148712 \\
.0171898 \\
1.124201 \\
3.185313\end{array}$ & $\begin{array}{l}.040038 \\
.0011649 \\
.7458806 \\
.0300852 \\
.7645041 \\
1.24692\end{array}$ & $\begin{array}{r}-0.14 \\
-6.57 \\
-0.69 \\
0.57 \\
1.47 \\
2.55\end{array}$ & $\begin{array}{l}0.892 \\
0.000 \\
0.490 \\
0.568 \\
0.141 \\
0.011\end{array}$ & $\begin{array}{r}-.0838851 \\
-.0099308 \\
-1.97677 \\
-.0417761 \\
-.3741998 \\
.7413954\end{array}$ & $\begin{array}{r}.073061 \\
-.0053646 \\
.9470278 \\
.0761557 \\
2.622601 \\
5.629231\end{array}$ \\
\hline $\begin{array}{r}\text { t i e mpo } \\
\text { cos to } \\
\text { gnero } \\
\text { edad } \\
\text { jef eh } \\
\text { _cons }\end{array}$ & $\begin{array}{r}-.0691814 \\
-.0039591 \\
.3917918 \\
.0034254 \\
.6346214 \\
3.651146\end{array}$ & $\begin{array}{l}.0303267 \\
.0007395 \\
.4653816 \\
.0222909 \\
.5125226 \\
1.012029\end{array}$ & $\begin{array}{r}-2.28 \\
-5.35 \\
0.84 \\
0.15 \\
1.24 \\
3.61\end{array}$ & $\begin{array}{l}0.023 \\
0.000 \\
0.400 \\
0.878 \\
0.216 \\
0.000\end{array}$ & $\begin{array}{r}-.1286207 \\
-.0054084 \\
-.5203394 \\
-.0402639 \\
-.3699044 \\
1.667607\end{array}$ & $\begin{array}{r}-.0097421 \\
-.0025098 \\
1.303923 \\
.0471147 \\
1.639147 \\
5.634686\end{array}$ \\
\hline $\begin{array}{r}\text { t i e mpo } \\
\text { cost o } \\
\text { gnero } \\
\text { edad } \\
\text { jefeh } \\
\text { _cons }\end{array}$ & $\begin{array}{r}-.2914236 \\
.0022856 \\
.5850338 \\
-.0128812 \\
1.141019 \\
.3095794\end{array}$ & $\begin{array}{l}.0504613 \\
.0007664 \\
4998418 \\
.0292172 \\
.5683027 \\
1.212272\end{array}$ & $\begin{array}{r}-5.78 \\
2.98 \\
1.17 \\
-0.44 \\
2.01 \\
0.26\end{array}$ & $\begin{array}{l}0.000 \\
0.003 \\
0.242 \\
0.659 \\
0.045 \\
0.798\end{array}$ & $\begin{array}{r}-.3903259 \\
.0007835 \\
-.394638 \\
-.0701458 \\
.0271665 \\
-2.066429\end{array}$ & $\begin{array}{r}-.1925213 \\
.0037877 \\
1.564706 \\
.0443834 \\
2.254872 \\
2.685588\end{array}$ \\
\hline $\begin{array}{l}\text { t i e mpo } \\
\text { cos to } \\
\text { gnero } \\
\text { edad } \\
\text { jef eh } \\
\text { _cons }\end{array}$ & $\begin{array}{r}-.4623022 \\
.0057679 \\
-.052248 \\
.0184227 \\
.2340466 \\
-7.902553\end{array}$ & $\begin{array}{l}.1286202 \\
.0013201 \\
1.651334 \\
.0735278 \\
1.891345 \\
3.431292\end{array}$ & $\begin{array}{r}-3.59 \\
4.37 \\
-0.03 \\
0.25 \\
0.12 \\
-2.30\end{array}$ & $\begin{array}{l}0.000 \\
0.000 \\
0.975 \\
0.802 \\
0.902 \\
0.021\end{array}$ & $\begin{array}{r}-.7143932 \\
.0031805 \\
-3.288803 \\
-.1256891 \\
-3.472922 \\
-14.62776\end{array}$ & $\begin{array}{r}-.2102112 \\
.0083552 \\
3.184307 \\
.1625346 \\
3.941016 \\
-1.177345\end{array}$ \\
\hline $\begin{array}{l}\text { t i e mpo } \\
\text { cos to } \\
\text { gnero } \\
\text { edad } \\
\text { jef eh } \\
\text { _cons }\end{array}$ & $\begin{array}{r}-.3921288 \\
.0061938 \\
1.314974 \\
.0218565 \\
-.7730957 \\
-10.14228\end{array}$ & $\begin{array}{r}.1256349 \\
.001337 \\
1.61598 \\
.0735986 \\
1.870107 \\
3.556456\end{array}$ & $\begin{array}{r}-3.12 \\
4.63 \\
0.81 \\
0.30 \\
-0.41 \\
-2.85\end{array}$ & $\begin{array}{l}0.002 \\
0.000 \\
0.416 \\
0.766 \\
0.679 \\
0.004\end{array}$ & $\begin{array}{r}-.6383687 \\
.0035734 \\
-1.852288 \\
-.1223941 \\
-4.438438 \\
-17.11281\end{array}$ & $\begin{array}{r}-.145889 \\
.0088143 \\
4.482236 \\
.1661071 \\
2.892247 \\
-3.171759\end{array}$ \\
\hline
\end{tabular}

( $\operatorname{modo}==5$ is the base out come) 